\title{
Linear stability analysis of rapid granular flow on a slope and density wave formation
}

\author{
By NAMIKO MITARAI AND HIIZU NAKANISHI \\ Department of Physics, Kyushu University 33, Fukuoka 812-8581, Japan
}

(Received 4 November 2018)

The linear stability of rapid granular flow on a slope under gravity against the longitudinal perturbation is analyzed using hydrodynamic equations. It is demonstrated that the steady flow uniform along the flow direction becomes unstable against the longwavelength perturbations longitudinal to the flow direction for certain parameter ranges to form the density wave, in contrast with the finite wavelength instability against the transverse perturbation (Forterre \& Pouliquen 2002). It is shown that the instability can be understood as the the long-wave instability of the kinematic waves in a quasi-one dimensional system. The results are compared with our previous molecular dynamics simulations (Mitarai \& Nakanishi 2001), where the spontaneous density wave formation has been found.

\section{Introduction}

Granular flow exhibits a variety of dynamical phenomena, which have been attracting research interests for years (for reviews, see e.g. Savage 1984 and Jaeger, Nagel \& Behringer 1996). Its complex behaviors can be seen even in a simple situation like the gravitational flow on a slope. When the inclination angle is large and the slope is rough, a rapid and relatively low-density flow is realized, and the interaction between grains is dominated by inelastic collisions. On the other hand, when the inclination angle is small, the flow becomes dense and slow, and the frictional interaction plays an important role (Savage 1984; Mitarai \& Nakanishi 2003). The comprehensive rheology of the granular flow has not been established yet, except for the rapid collisional flow regime, where the hydrodynamic models have been developed with the constitutive relations based on the kinetic theory of inelastic hard spheres (Jenkins \& Savage 1983; Campbell 1990; Lun et al.1984; Goldhirsh 2003); it has been demonstrated that a certain quantitative agreement can be achieved for the steady flow by introducing the spinning motion of each grains (Mitarai, Hayakawa \& Nakanishi 2002). The steady granular flow, however, turns out to be unstable in various ways, and shows rich phenomena.

In the experiment on a shallow granular flow on a wide slope, Forterre \& Pouliquen(2001) have observed that there appears a regular pattern of longitudinal streaks along the flowing direction. This phenomenon has been analyzed by means of the hydrodynamic equations for rapid granular flow (Forterre \& Pouliquen 2002). They have calculated the steady solutions and examined its linear stability numerically. They have found that, at a certain parameter region, the steady flow shows the "inverted density profiles", in which the maximum density appears not at the bottom but at a finite distance from the bottom because of the agitation by the collisions with the rough solid bottom. It has been shown that the solutions with the "inverted density profiles" are unstable against the perturbations transverse to the flowing direction, and the instability results in the 
vortex patterns analogous to the rolls in the Rayleigh-Bérnard convection; the streaks found in the experiment were interpreted as the result of the rolls of vortices.

Another instability that has been observed is the density wave formation along the flowing direction; experimentally, Louge \& Keast(2001) have observed the jamming patters traveling upstream in the dense chute flow, and Prasad, Pal \& Römkens (2000) have found the waves develops in the shallow flow as they travel downstream. The present authors have performed the molecular dynamics simulations of two-dimensional granular flow on a slope and found that the steady flows are unstable against the density wave formation when the length of the slope is long enough and/or the particle density is low enough (Mitarai \& Nakanishi 2001).

The purpose of this paper is to perform the linear stability analysis on the hydrodynamic equations to investigate the nature of the density wave formation instability found in the experiments and the numerical simulations. The basic method is the same with that used in Forterre \& Pouliquen(2002), but we examine the stability against the perturbations longitudinal to the flowing direction in contrast with the case of Forterre \& Pouliquen(2002), where the transverse stability has been studied.

This paper is organized as follows. In $\$ 2$ the hydrodynamic model for rapid granular flow is introduced. The steady solutions uniform along the flow direction are numerically obtained in $\$ 3$ and the results of the linear stability analysis is presented in 4 In discussions and the comparison with the molecular dynamics simulations are given. The results are summarized in $\$ 6$

\section{Hydrodynamic Equations for Granular Flows}

\subsection{Hydrodynamic equations and constitutive relations}

The hydrodynamic fields for granular flows in three dimensions are the mass density $\rho$, the mean velocity $\boldsymbol{u}$, and the granular temperature $T$, where $T=<\delta \boldsymbol{u}^{2}>/ 3$. Here, $\delta \boldsymbol{u}=\boldsymbol{u}-\langle\boldsymbol{u}\rangle$ and $\langle\ldots\rangle$ represents the average over the microscopic scale. Under gravity, they follow

$$
\begin{aligned}
\left(\frac{\partial}{\partial t}+\boldsymbol{u} \cdot \nabla\right) \rho & =-\rho \nabla \cdot \boldsymbol{u}, \\
\rho\left(\frac{\partial}{\partial t}+\boldsymbol{u} \cdot \nabla\right) \boldsymbol{u} & =\rho \boldsymbol{g}-\nabla \cdot \Sigma, \\
\frac{3}{2} \rho\left(\frac{\partial}{\partial t}+\boldsymbol{u} \cdot \nabla\right) T & =-\nabla \cdot \boldsymbol{q}-\Sigma: \nabla \boldsymbol{u}-\Gamma,
\end{aligned}
$$

with the acceleration of gravity $\boldsymbol{g}$, the stress tensor $\Sigma$, the heat flux $\boldsymbol{q}$, and the energy loss $\Gamma$ due to the inelastic nature of interactions between grains.

We employ the constitutive relations derived by Lun et al.(1984) for three dimensional system based on the kinetic theory of the inelastic particles: $\dagger$

$$
\begin{aligned}
\Sigma & =(p-\zeta \nabla \cdot \boldsymbol{u}) I-2 \mu S, \\
\boldsymbol{q} & =-\kappa \nabla T,
\end{aligned}
$$

$\dagger$ The original form of $\boldsymbol{q}$ derived by Lun et al.(1984) is $\boldsymbol{q}=-\kappa \nabla T-\kappa_{h} \nabla \nu$. The coefficient $\kappa_{h}$ is proportional to $\left(1-e_{p}\right)$, thus disappears in the elastic limit. We checked that the influence of the term $\kappa_{h} \nabla \nu$ on the steady solutions is small in the considered parameter region, therefore neglected this term as Forterre \& Pouliquen(2002) 


$$
\begin{aligned}
& f_{1}(\nu)=\nu\left(1+4 \eta \nu g_{0}(\nu)\right) \\
& f_{2}(\nu)=\frac{5 \pi^{1 / 2}}{96 \eta(2-\eta)}\left(1+\frac{8}{5} \eta \nu g_{0}(\nu)\right)\left(\frac{1}{g_{0}(\nu)}+\frac{8}{5} \eta(3 \eta-2) \nu\right)+\frac{8}{5 \pi^{1 / 2}} \eta \nu^{2} g_{0}(\nu) \\
& f_{3}(\nu)=\frac{8}{3 \pi^{1 / 2}} \eta \nu^{2} g_{0}(\nu) \\
& f_{4}(\nu)=\frac{25 \pi^{1 / 2}}{16 \eta(41-33 \eta)}\left(1+\frac{12}{5} \eta \nu g_{0}(\nu)\right)\left(\frac{1}{g_{0}(\nu)}+\frac{12}{5} \eta^{2}(4 \eta-3) \nu\right)+\frac{4}{\pi^{1 / 2}} \eta \nu^{2} g_{0}(\nu) \\
& f_{5}(\nu)=\frac{12}{\pi^{1 / 2}} \nu^{2} g_{0}(\nu) \\
& f_{6}(\nu)=\frac{\sqrt{3} \pi \nu g_{0}(\nu)}{2 \nu_{m} f_{4}(\nu)} \\
& f_{7}(\nu)=\frac{\pi \nu g_{0}(\nu)}{2 \sqrt{3} \nu_{m} f_{2}(\nu)}
\end{aligned}
$$

TABle 1. Dimensionless functions used in the constitutive relations and the boundary conditions with $\eta \equiv\left(1+e_{p}\right) / 2$.

where

$$
S=\frac{1}{2}\left[\nabla \boldsymbol{u}+(\nabla \boldsymbol{u})^{t}\right]-\frac{1}{3}(\nabla \cdot \boldsymbol{u}) I
$$

and

$$
\begin{aligned}
& p(\nu, T)=\rho_{p} f_{1}(\nu) T, \quad \mu(\nu, T)=\rho_{p} \sigma f_{2}(\nu) T^{1 / 2}, \quad \zeta(\nu, T)=\rho_{p} \sigma f_{3}(\nu) T^{1 / 2}, \\
& \kappa(\nu, T)=\rho_{p} \sigma f_{4}(\nu) T^{1 / 2}, \quad \Gamma(\nu, T)=\frac{\rho_{p}}{\sigma}\left(1-e_{p}^{2}\right) f_{5}(\nu) T^{3 / 2}
\end{aligned}
$$

with the material density of particle $\rho_{p}$, the packing fraction $\nu=\rho / \rho_{p}$, the particle diameter $\sigma$, and the restitution coefficient between particles $e_{p}$. Here, $I$ represents the unit matrix. The dimensionless functions $f_{i}(\nu)(i=1, \ldots, 5)$ are given in Table 11 For the radial distribution function $g_{0}(\nu)$ in these functions, we adopted the form suggested by Lun \& Savage(1986)

$$
g_{0}(\nu)=\frac{1}{\left(1-\nu / \nu_{m}\right)^{2.5 \nu_{m}}}
$$

with the maximum solid fraction $\nu_{m}$, for which we use 0.60 as Forterre \& Pouliquen(2002)

In the following, all the variables are non-dimensionalized by the length unit $\sigma$, the mass unit $\rho_{p} \sigma^{3}$, and the time unit $\sqrt{\sigma / g}$. The density field is expressed by the packing fraction $\nu$ instead of the mass density $\rho$. The restitution coefficient between particles is set to be $e_{p}=0.7$, that is the value used in our previous simulations (Mitarai \& Nakanishi 2001).

\subsection{Boundary conditions}

The granular flow has a non-zero slip velocity at the solid boundary, where we should impose the momentum and the kinetic energy balances. Sophisticated boundary conditions have been proposed based on microscopic calculations of the kinetic theory for specific geometries (Jenkins \& Richman 1986; Richman 1988; Jenkins 1992). We employ, 
however, a simpler form of the boundary condition obtained from a heuristic approach (Johnson \& Jackson 1987; Johnson, Nott \& Jackson 1987);

$$
\begin{aligned}
-\boldsymbol{n} \cdot \Sigma \cdot \boldsymbol{t} & =\eta^{*}(\nu, T)\left|\boldsymbol{u}_{s}\right|, \\
\boldsymbol{n} \cdot \boldsymbol{q} & =-\boldsymbol{u}_{s} \cdot \Sigma \cdot \boldsymbol{n}-\Gamma^{*}(\nu, T),
\end{aligned}
$$

where the unit vector $\boldsymbol{n}$ is normal to the floor, $\boldsymbol{u}_{s}$ is the slip velocity at the floor, and $\boldsymbol{t}=\boldsymbol{u}_{s} /\left|\boldsymbol{u}_{s}\right|$ is the unit vector along the slip velocity.

The first equation (2.9) expresses that the stress at the boundary balances with the momentum transfer due to the collisions between the slope and the flowing grains. The momentum transfer, or RHS of (2.9), is assumed to be given by

$$
\eta^{*}(\nu, T)\left|\boldsymbol{u}_{s}\right|=\frac{\pi}{6} \phi\left|\boldsymbol{u}_{s}\right| \Omega(\nu, T),
$$

with the collision rate $\Omega(\nu, T)$ per unit time per unit area. Here, the factor $\pi / 6$ comes from the non-dimensionalization of the particle mass $m=\sigma^{3} \pi / 6$. The parameter $\phi$ characterizes the roughness of the boundary, and the expression means that the fraction $\phi$ of the particle momentum is transfered to the boundary in each collision, therefore, the larger value of $\phi$ represents the rougher boundary. For the rough boundary in the two-dimensional system with closed packed disks, Jenkins \& Richman(1986) estimated as $\phi \approx 0.1$, but Forterre \& Pouliquen(2002) adopted the smaller value $\phi=0.05$ for most of the cases because they expected that a boundary with closed packed spheres in threedimensional system is smoother on average. In this paper, we mainly use $\phi=0.05$, but the case of $\phi=0.10$ is also examined in order to see general tendencies.

The second equation (2.10) represents the energy balance, and means that the heat flux at the boundary comes from two effects, namely, the frictional heating due to the non-zero slip velocity and the energy loss due to the inelastic collision with the floor. The energy loss term $\Gamma^{*}$ in (2.10) is given by

$$
\Gamma^{*}=\Phi \cdot \frac{\pi}{6} \cdot \frac{3}{2} T \cdot \Omega(\nu, T)
$$

with the collision rate $\Omega(\nu, T)$. The parameter $\Phi$ represents the rate of energy loss per collision $\dagger$, and we use $\Phi=0.39$ in this paper.

For the collision rate $\Omega(\nu, T)$, we use the form adopted by Forterre \& Pouliquen(2002) i.e., $\Omega(\nu, T)=\sqrt{3 T} \nu g_{0}(\nu) / \nu_{m}$ : then the expression of $\eta^{*}(\nu, T)$ and $\Gamma^{*}(\nu, T)$ in nondimensionalized form are

$$
\begin{aligned}
\eta^{*}(\nu, T) & =\phi f_{7}(\nu) f_{2}(\nu) T^{1 / 2}, \\
\Gamma^{*}(\nu, T) & =\frac{1}{2} \Phi f_{6}(\nu) f_{4}(\nu) T^{3 / 2},
\end{aligned}
$$

where the dimensionless functions $f_{6}(\nu)$ and $f_{7}(\nu)$ are given in Table 1

At infinity, we impose the condition that the stress and the heat flux vanish, namely,

$$
\Sigma \rightarrow 0 \text { and } \boldsymbol{q} \rightarrow 0 \quad \text { as } \quad y \rightarrow \infty
$$

where the $y$ axis is taken perpendicular to the floor (figure 1).

$\dagger$ Johnson \& Jackson(1987) explicitly relate $\Phi$ to the restitution coefficient between the floor and the particles $e_{w}$ in the form $\Phi=\left(1-e_{w}^{2}\right)$, but we adopt 2.12) as a more general expression. 


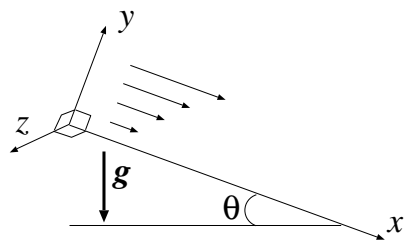

Figure 1. The coordinate system.

\section{Steady Flows}

\subsection{Equations and Numerical method}

First, we consider the steady solution which is uniform along the slope for (2.1) - (2.3) with the boundary conditions (2.9), (2.10), and (2.15) in the form

$$
\begin{aligned}
\nu(x, y, z, t) & =\nu_{0}(y), \\
\boldsymbol{u}(x, y, z, t) & =\left(u_{0}(y), 0,0\right), \\
T(x, y, z, t) & =T_{0}(y),
\end{aligned}
$$

where we take the $x$ axis along the slope, the $y$ axis perpendicular to the floor, and the $z$ axis perpendicular to the $x-y$ plane (figure 1).

Then, the equations (2.1) - (2.3) are written as

$$
\begin{aligned}
& 0=\nu_{0} \sin \theta-\frac{\mathrm{d} \Sigma_{x y}^{0}}{\mathrm{~d} y}, \\
& 0=-\nu_{0} \cos \theta-\frac{\mathrm{d} \Sigma_{y y}^{0}}{\mathrm{~d} y}, \\
& 0=-\Sigma_{x y}^{0} \frac{\mathrm{d} u_{0}}{\mathrm{~d} y}-\frac{\mathrm{d} q_{y}^{0}}{\mathrm{~d} y}-\Gamma^{0},
\end{aligned}
$$

where the superscript 0 denotes that the functions are for the steady solution, namely, $\boldsymbol{q}^{0}=-\kappa\left(\nu_{0}, T_{0}\right) \nabla T_{0}=\left(0, q_{y}^{0}, 0\right)$, etc. By integrating (3.4) and (3.5) over $y$ with the stress free condition at infinity, we obtain the condition

$$
\Sigma_{x y}^{0}=-\tan \theta \Sigma_{y y}^{0} .
$$

From (3.4)-(3.7) and the constitutive relations, we have

$$
\begin{aligned}
\nu_{0}^{\prime}(y) & =-\frac{f_{1}^{0} T_{0}^{\prime}+\nu_{0} \cos \theta}{f_{1, \nu}^{0} T_{0}}, \\
u_{0}^{\prime}(y) & =\frac{f_{1}^{0} T_{0}^{1 / 2}}{f_{2}^{0}} \tan \theta, \\
T_{0}^{\prime \prime}(y) & =\frac{1}{f_{4}^{0}}\left[\left(1-e_{p}^{2}\right) f_{5}^{0} T_{0}-f_{2}^{0} u_{0}^{\prime}-f_{4, \nu}^{0} \nu_{0}^{\prime} T_{0}^{\prime}-\frac{\left(T_{0}^{\prime}\right)^{2}}{2 T_{0}} f_{4}^{0}\right],
\end{aligned}
$$

where $f_{i}^{0} \equiv f_{i}\left(\nu_{0}\right),\left.f_{i, \nu}^{0} \equiv \frac{\mathrm{d}}{\mathrm{d} \nu} f_{i}(\nu)\right|_{\nu=\nu_{0}}$, and the prime indicates the derivative by its argument.

The boundary conditions (2.9) and (2.10) at the floor $(y=0)$ for the steady solution can be written as

$$
T_{0}=\left(\frac{\phi f_{2}^{0} f_{7}^{0}}{f_{1}^{0} \tan \theta} u_{0}\right)^{2}
$$


6

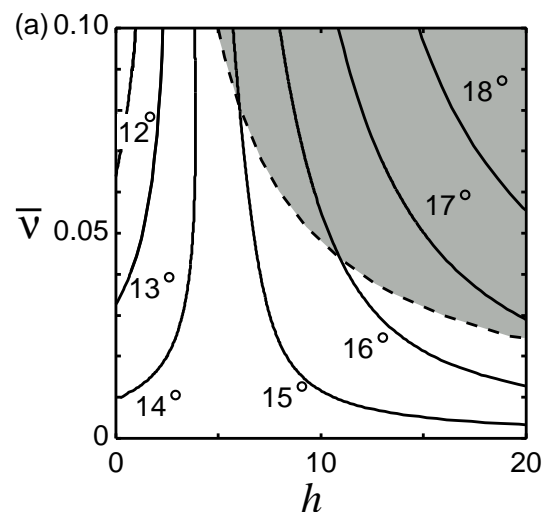

N. Mitarai and H. Nakanishi

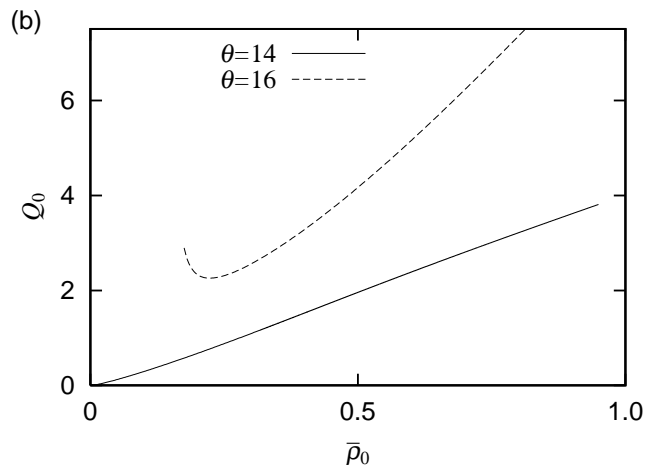

Figure 2. (a)The contour lines of $\theta$ in the $(h, \bar{\nu})$ plane for $\phi=0.05$. The region of the non-monotonic density profiles is shown by a grey region. (b)The flux $Q_{0}$ vs the one-dimensional density $\bar{\rho}_{0}$ for $\phi=0.05$. The solid line and the dashed line are for $\theta=14^{\circ}$ and $\theta=16^{\circ}$, respectively.

$$
T_{0}^{\prime}=-f_{6}^{0}\left(\frac{1}{3} \phi u_{0}^{2}-\frac{1}{2} \Phi T_{0}\right) .
$$

The boundary condition (2.15) that the stress and the energy flux should vanish at infinity is satisfied when (Ahn, Brennen \& Sabersky 1992)

$$
T_{0}^{\prime}(y) \rightarrow 0 \quad \text { when } \quad y \rightarrow \infty .
$$

In order to obtain the steady solutions, we integrate (3.8), (3.9), and (3.10) numerically using the fourth-order Runge-Kutta method with the boundary conditions (3.11) and (3.12). We employ the shooting method to find the solution which satisfies the condition (3.13) (Forterre \& Pouliquen 2002); for a given inclination angle $\theta$ and a given density at the floor $\nu_{0}(0)$, we search for a solution by adjusting the value of the velocity at the floor $u_{0}(0)$. In the actual calculations, we integrate the equations numerically from $y=0$ to a certain height $y_{\max }$, and search for the solution which gives $\left|T_{0}^{\prime}\left(y_{\max }\right)\right|<10^{-7}$. The value of $y_{\max }$ is chosen to be large enough in comparison with the relaxation length, that depends on the parameters and can be determined only after the solution is obtained.

We use $\theta$ and $\nu_{0}(0)$ to specify the solution in the rest of the paper.

\subsection{Numerical solutions}

For a given roughness $\phi$ of the slope, steady solutions are found for a certain range of the inclination angle of the slope $\theta$ (Forterre \& Pouliquen 2002). We present the steady solutions for the two cases, (i) $\phi=0.05$ and (ii) $\phi=0.10$; the most of the results are for the case of $\phi=0.05$, and the case of $\phi=0.10$ will be given to examine general trend.

\subsubsection{The case of $\phi=0.05$}

For an appropriate $\theta$, there exist steady solutions for a given density at the floor $\nu_{0}(0)$. We numerically find the steady solution for the range $8^{\circ} \lesssim \theta \lesssim 20^{\circ}$ for the moderate density; towards the lower limit of $\theta$, the length scale of the density decay in the $y$-direction tends to zero and becomes smaller than the particle diameter, which is physically unacceptable, while the decay length of density diverges towards the upper limit of $\theta$. This is consistent with the analysis by Anderson \& Jackson(1992) that the steady solution in the high-density limit is allowed for a finite range of $\theta$.

For a given steady solution, we define the "thickness" $h$ and the "mean density" $\bar{\nu}$ : the 
(a)

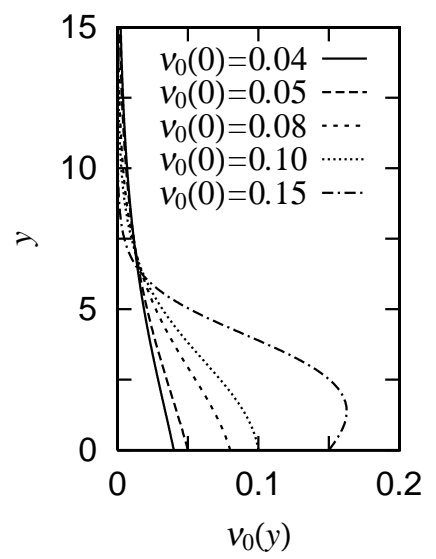

(b)

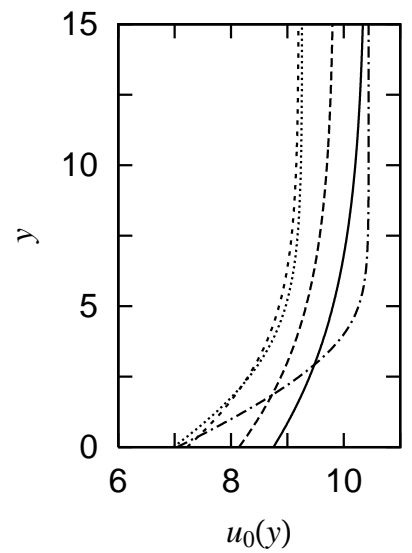

(c)

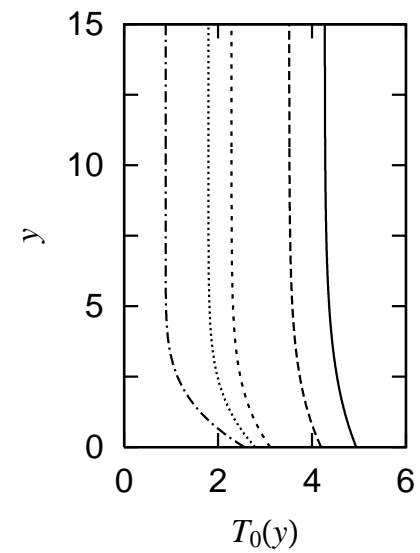

Figure 3. The steady solutions for $\phi=0.05$ and $\theta=16^{\circ}$; the density profiles (a), the velocity profiles (b), and the temperature profiles (c). Different line types correspond to the solutions with different densities; $\nu_{0}(0)=0.04$ (solid lines), 0.05 (long-dashed lines), 0.08 (short-dashed lines), 0.10 (dotted lines), and 0.15 (dash-dotted lines).

thickness $h$ is the value of $y$ where the density is $1 \%$ of the maximum density, and the mean density $\bar{\nu}$ is given by

$$
\bar{\nu}=\frac{1}{h} \int_{0}^{\infty} \nu_{0}(y) \mathrm{d} y .
$$

It has been found numerically by Forterre \& Pouliquen(2002) that there exists a one to one correspondence between $\left(\theta, \nu_{0}(0)\right)$ and $(h, \bar{\nu})$.

Figure 2(a) shows the contour lines of $\theta$ in the $(h, \bar{\nu})$ plane, where $\theta$ increases from left to right. In this plot, it is clear that $h$ goes to zero as $\theta$ decreases and $h$ diverges as $\theta$ increases. There is a separatrix near the bottom at $h \approx 7$, where the value of $\theta$ is around $15^{\circ}$; the contour line for $\theta \leqslant 14^{\circ}$ leads to $h=0$ as $\bar{\nu}$ decreases, while $h$ diverges along the contour lines for $\theta \geqslant 15^{\circ}$ as $\bar{\nu}$ becomes small.

The difference between $\theta \geqslant 15^{\circ}$ and $\theta \leqslant 14^{\circ}$ can be seen in the relation between the flux $Q_{0}$ and the one-dimensional density $\bar{\rho}_{0}$ defined as

$$
Q_{0} \equiv \int_{0}^{\infty} \nu_{0}(y) u_{0}(y) \mathrm{d} y \quad \text { and } \quad \bar{\rho}_{0} \equiv \int_{0}^{\infty} \nu_{0}(y) \mathrm{d} y,
$$

respectively, for a fixed inclination angle. It is found that $Q_{0}$ is an increasing function of $\bar{\rho}_{0}$ for $\theta \leqslant 14^{\circ}$, while $Q_{0}$ has a minimum at a finite $\bar{\rho}_{0}$ for $\theta \geqslant 15^{\circ}$; the plot of $Q_{0}$ vs $\rho_{0}$ is shown in figure 2(b) for $\theta=14^{\circ}$ and $\theta=16^{\circ}$.

The typical profiles of the density, the velocity, and the temperature for $\theta=16^{\circ}$ are shown in figure 3 for the density at the floor $\nu_{0}(0)=0.04 \sim 0.15$. We see in figure 3(a) that the density decays monotonically when the density at the floor $\nu_{0}(0)$ is small enough $\left(\nu_{0}(0) \leqslant 0.10\right)$, while for higher density $\left(\nu_{0}(0)=0.15\right)$ the maximum density appears at a finite height. The region where the maximum density appears at a finite height is shown in figure 2 (a) as a grey region. We focus on the lower density region because the density decays monotonically in our previous simulation (Mitarai \& Nakanishi 2001; Mitarai et al.2002).

For $\nu_{0}(0) \lesssim 0.10$, the higher density flow shows lower flowing speed in the case of $\theta=16^{\circ}$, which results in the decrease of the flux $Q_{0}$ as $\bar{\rho}_{0}$ increase for $\bar{\rho}_{0} \lesssim 0.2$ (figure 2(b)). For higher density $\left(\nu_{0}(0)=0.15\right.$ in figure 3), the flowing speed gets faster with $\bar{\rho}_{0}$, 

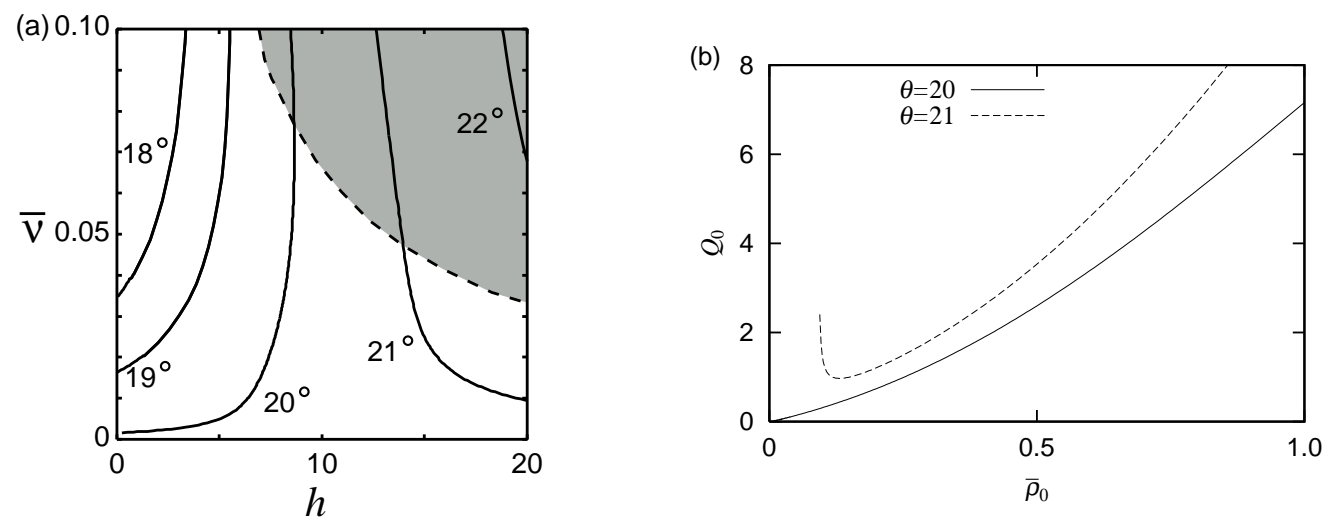

FiguRE 4. (a)The contour lines of $\theta$ in the $(h, \bar{\nu})$ plane for $\phi=0.10$. The region of non-monotonic density profiles is shown by a grey region. (b)Relation between $Q_{0}$ vs $\bar{\rho}_{0}$ for $\phi=0.10$. The solid line and the dashed line are for $\theta=20^{\circ}$ and $\theta=21^{\circ}$, respectively.

(a)

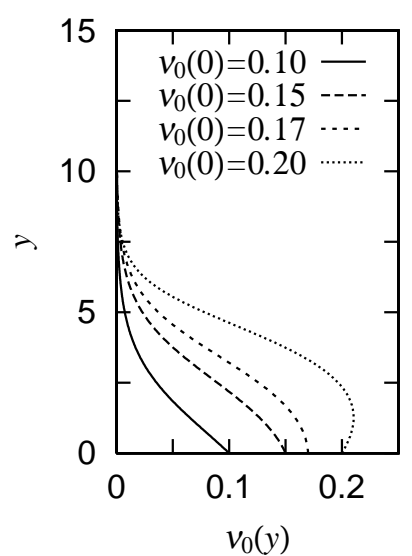

(b)

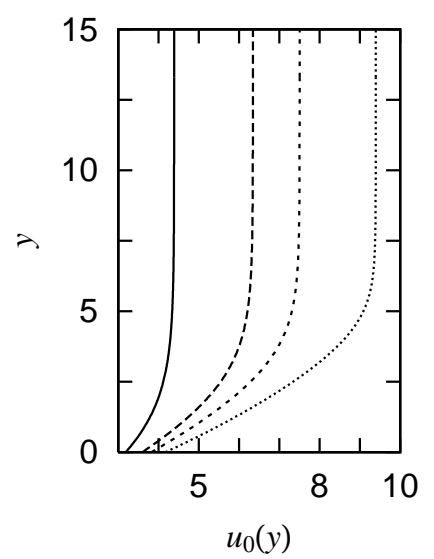

(c)

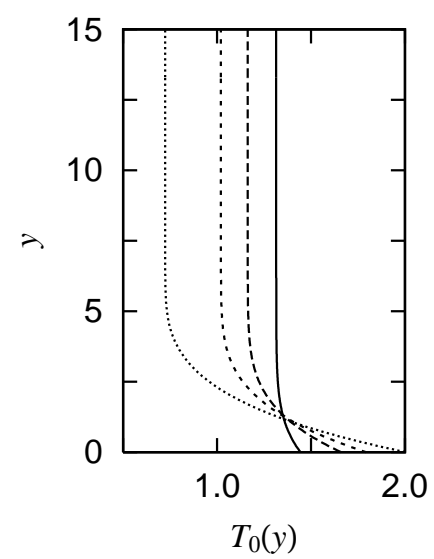

Figure 5. The steady solutions for $\phi=0.10$ and $\theta=20^{\circ}$. The density profiles (a), the velocity profiles (b), and the temperature profiles (c). Different line types correspond to the solutions with different densities; $\nu_{0}(0)=0.10$ (solid lines), 0.15 (long-dashed lines) 0.17 (short-dashed lines), and 0.20 (dotted lines).

which causes the increase of the flux $Q_{0}$ for higher $\bar{\rho}_{0}$. As a result, $Q_{0}$ has a minimum at a finite density.

In the case of $\theta \leqslant 14^{\circ}$, the velocity continuously decrease as the density becomes lower, and $Q_{0}$ becomes a increasing function of $\bar{\rho}_{0}$.

\subsubsection{The case of $\phi=0.10$}

The slope is rougher than the previous case, and the steady solution exists for $12^{\circ} \lesssim$ $\theta \lesssim 25^{\circ}$. The contour lines for $\theta$ in the $(h, \bar{\nu})$ plane are shown in figure प(a). As in the case of $\phi=0.05, h$ goes to zero as density becomes lower for smaller $\theta$, while $h$ diverges for smaller density when $\theta$ is large enough. Figure 4 (b) shows $\bar{\rho}_{0}$ dependence of $Q_{0}$, which is a monotonically increasing function for $\theta=20^{\circ}$ and has a minimum for $\theta=21^{\circ}$. The typical solutions are shown for $\theta=20^{\circ}$ in figure 5 For large enough $h$ and $\bar{\nu}$, the maximum density appears at a finite distance from the floor. The region of the non-monotonic density profile is shown by a grey region in figure 4 (a). 


\section{Linear Stability Analysis; Density Wave Formation}

\subsection{Normal mode analysis}

We restrict our stability analysis to the perturbation uniform along the $z$ direction, because we are interested in the instability along the flow direction. The flow is perturbed around the steady solution as

$$
\begin{aligned}
\nu(x, y, t) & =\nu_{0}(y)+\nu_{1}(x, y, t), \\
\boldsymbol{u}(x, y, t) & \left.=\left(u_{0}(y), 0,0\right)+\left(u_{1}(x, y, t), v_{1}(x, y, t)\right), 0\right), \\
T(x, y, t) & =T_{0}(y)+T_{1}(x, y, t) .
\end{aligned}
$$

The governing equations and the boundary conditions are linearized with respect to the deviations $\nu_{1}, u_{1}, v_{1}$, and $T_{1}$, but the resulting expressions are rather lengthy and given in Appendix A.

Now we look for the normal modes for the density, the velocity, and the temperature perturbations of the form

$$
\left(\nu_{1}, u_{1}, v_{1}, T_{1}\right)=\operatorname{Re}[(\hat{\nu}(y), \hat{u}(y), \hat{v}(y), \hat{T}(y)) \exp (\alpha t+i k x)] .
$$

The flow is linearly unstable if $\operatorname{Re}(\alpha)>0$.

As for the boundary condition at the free surface, the asymptotic behavior of the perturbations at large $y$ are used. When $y$ is much larger than the decay length of the density and thus $\nu_{0}(y)$ is very small, the density perturbation should also decay $\left(\hat{\nu} \propto \nu_{0}\right)$, and $\hat{u}, \hat{v}$, and $\hat{T}$ decay proportional to $\exp (-k y)$ (Forterre \& Pouliquen 2002), therefore, we imposed the boundary condition that

$$
\hat{u}^{\prime}(y)=-k \hat{u}(y), \quad \hat{v}^{\prime}(y)=-k \hat{v}(y), \quad \hat{T}^{\prime}(y)=-k \hat{T}(y),
$$

at $y=y_{\max } ; y_{\max }$ is a large enough height where $\nu_{0}\left(y_{\max }\right)<10^{-9}$, in addition to the condition $\left|T_{0}^{\prime}\left(y_{\max }\right)\right|<10^{-7}$ discussed in 3

We solve the eigenvalue problems of the linearized equations (A 1)- A 4 for (4.4 numerically using the Chebychev collocation method with the discretization in the $y$ direction (Gottilieb, Hussaini \& Orsag 1984; Canuto et al.1988; Boyd 2001; Foterre \& Pouliquen 2002). It is known that the straightforward discretization of space requires two extra boundary conditions (Malik 1990; Foterre \& Pouliquen 2002), for which we use the momentum balance condition in the $y$ direction at $y=0$ and the decay condition for the density perturbation, i.e., $\hat{\nu}^{\prime}(y)=-\left(\cos \theta / T_{0}(y)\right) \hat{\nu}(y)$ at $y=y_{\max }$. In actual numerics to solve the generalized eigenvalue problem in the form $A \boldsymbol{V}=\alpha B \boldsymbol{V}$ for the complex eigenvalues $\alpha$ and the eigenvectors $\boldsymbol{V}$, we used LAPACK version 3.0 (Anderson et al.1999). The number of discretization $N_{d}$ is about 100 .

The numerically obtained eigenmodes contains unphysical modes, called spurious modes, due to the discretization (Mayer \& Powell 1992; Boyd 2001; Forterre \& Pouliquen 2002). For the spurious modes, it is known that the Chebychev coefficients of higher wave number are large, and the eigenvalues are sensitive to small change of $N_{d}$. We determine the eigenmodes as physical ones by checking that their Chebychev coefficients for higher wave number are small and their eigenvalues varies little upon changing $N_{d}$ : We confirmed that, for these modes, the highest ten coefficients are less than $10^{-7}$ when the eigenvectors are normalized so that the sum of the absolute values of the real part and the imaginary part of the largest component becomes one, and the variation of the eigenvalues by the small change of $N_{d}$ are less than $10^{-7}$. 


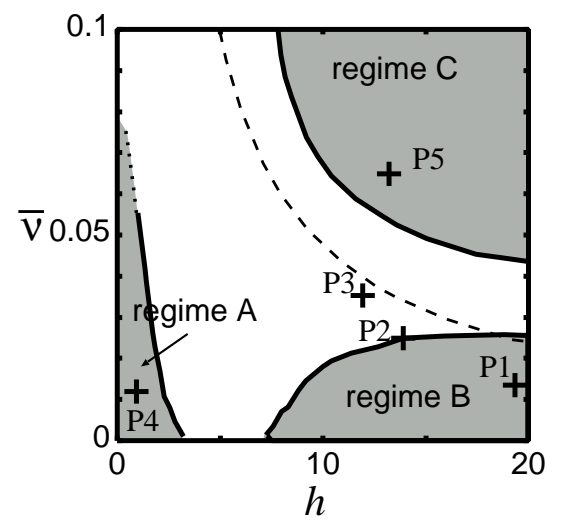

FiguRE 6 . The stability diagram for $h$ vs $\bar{\nu}$ for $\phi=0.05$, where the unstable (stable) regimes are shown by grey (white) regions. We find three unstable regimes $\mathrm{A}, \mathrm{B}$, and $\mathrm{C}$. The dashed line shows the boundary between the region of the non-monotonic density profiles and that of the monotonic density profiles (see also figure 21. The dispersion relations at the points P1 P5 are shown in figures 7 $\sim 9$ The region of $h<1$ where the stability boundary is shown by a dotted line was not examined in detail because the flow with the decay length less than the particle diameter is physically unacceptable.

(a)

$\operatorname{Re}(\alpha)$

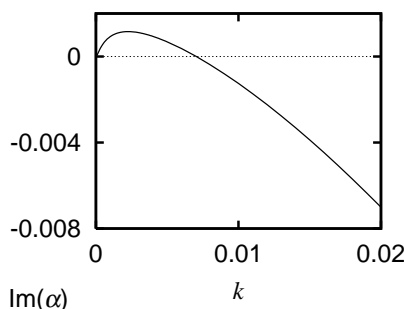

$\operatorname{Im}(\alpha)$

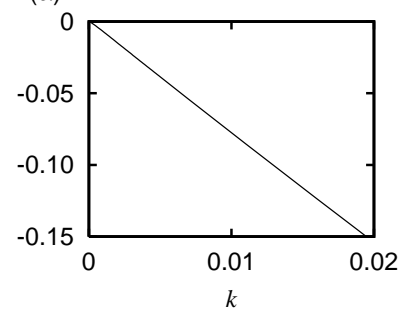

(b)

$\operatorname{Re}(\alpha)$

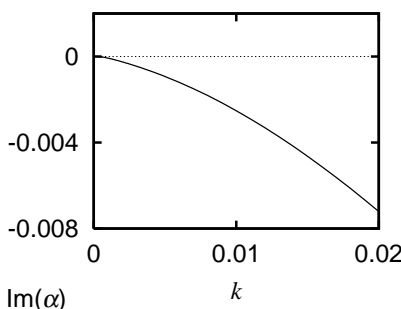

$\operatorname{lm}(\alpha)$

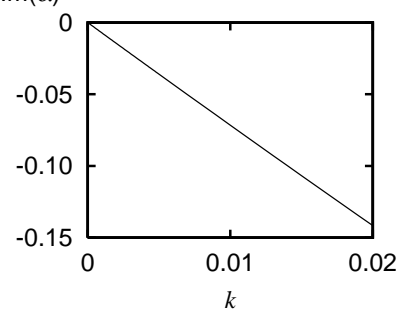

(c)

$\operatorname{Re}(\alpha)$

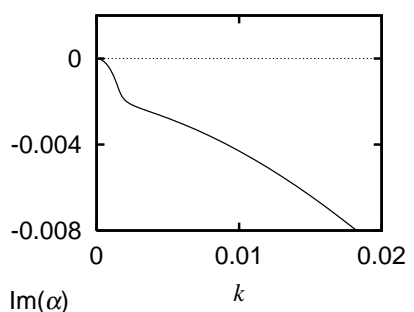

$\operatorname{Im}(\alpha)$

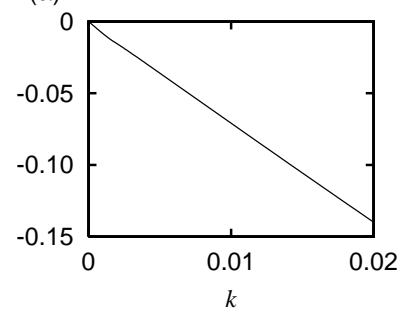

FiguRE 7. The dispersion relations of the least stable mode for the steady solutions with $\phi=0.05$ and $\theta=16^{\circ}$ for $\nu_{0}(0)=0.05(\mathrm{a}), 0.08(\mathrm{~b})$, and $0.10(\mathrm{c})$, which correspond to the points P1, P2, and $\mathrm{P} 3$ in figure 6 respectively.

\subsection{Stability diagram, dispersion relations, and eigenfunctions}

We present the results of the linear stability analysis in the cases of $\phi=0.05$ and $\phi=0.10$, for which the steady solutions are shown in $\$ 3.2$

\subsubsection{The case of $\phi=0.05$}

The stability diagram is shown in figure [in the parameter space of $h$ vs $\bar{\nu}$. The unstable (stable) regimes are shown by grey (white) regions, and the boundary between the region of the monotonic density profiles and that of the non-monotonic density profiles is shown by a dashed line. Within the investigated region, we find three unstable regimes; the 

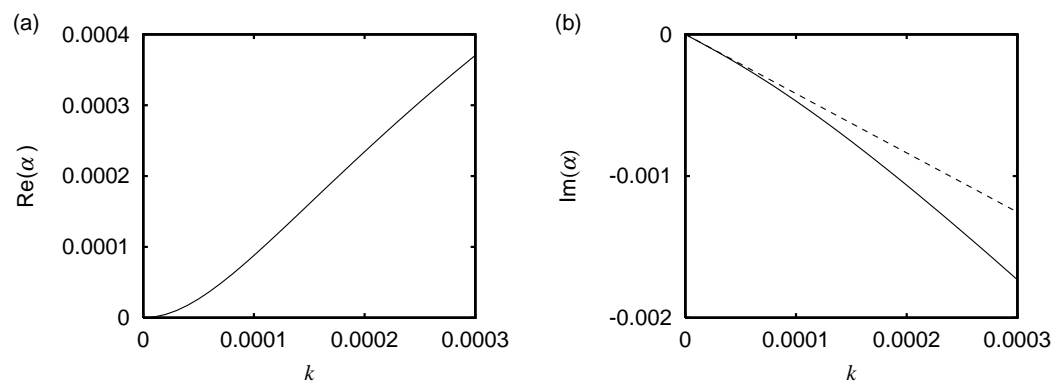

FiguRE 8 . The magnification of the dispersion relations near $k=0$ of the unstable stable mode for the steady solutions with $\phi=0.05$ and $\theta=16^{\circ}$ for $\nu_{0}(0)=0.05$ at $\mathrm{P} 1$ in the regime $\mathrm{B}$. $\operatorname{Re}[\alpha]$ grows quadratically in $k$, while the slope of $\operatorname{Im}[\alpha]$ is given by $-\mathrm{d} Q_{0} / \mathrm{d} \bar{\rho}_{0}$ in the long-wavelength limit, which is shown by a dashed line.

(a)
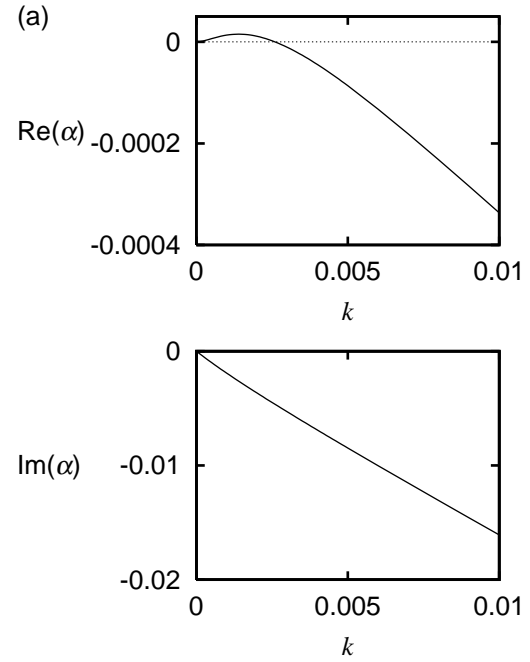

(b)
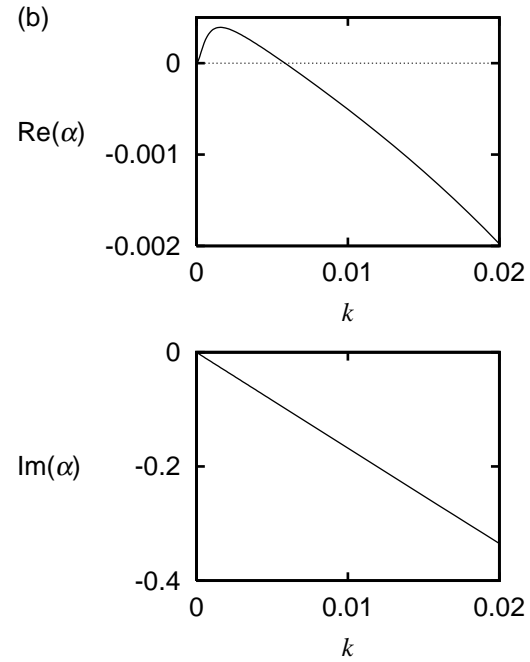

Figure 9. The dispersion relations of the unstable mode for $\phi=0.05$ with (a) $\theta=14^{\circ}$ and $\nu_{0}(0)=0.05(\mathrm{P} 4$; in regime $\mathrm{A})$ and $(\mathrm{b}) \theta=17^{\circ}$ and $\nu_{0}(0)=0.10(\mathrm{P} 5$; in regime $\mathrm{C})$.

regime $\mathrm{A}$ at small $h$ and small $\bar{\nu}$ region, the regime $\mathrm{B}$ at large $h$ and small $\bar{\nu}$ region, and the regime $\mathrm{C}$ at large $h$ and large $\bar{\nu}$ region. When we decrease the density with a constant inclination angle (along a contour in figure 2), eventually we will encounter either the regime $\mathrm{A}$ or the regime $\mathrm{B}$, namely, the flow with low enough density is always unstable. The two regimes are different in the steady flow behavior as we have seen already: in the regime $\mathrm{A}$ at the small $h$ side, the flow becomes slower as the density becomes smaller, while the flow in the regime B at the large $h$ side flows down faster for the smaller density. On the other hand, the denser flow can be unstable in the regime $\mathrm{C}$, which lies within the region of the non-monotonic density profiles. In this regime, the denser flow goes faster as in the regime $\mathrm{A}$.

The dispersion relations of the least stable modes $\alpha=\alpha(k)$ are shown in figure $\mathbf{7}$ for $\theta=16^{\circ}$ and $\nu_{0}(0)=0.05(\mathrm{a}), 0.08(\mathrm{~b})$, and $0.10(\mathrm{c})$, which correspond to the points $\mathrm{P} 1$, P2, and P3, respectively, in the stability diagram of figure 6 P1 lies within the unstable regime B. In all cases, it is found that the least stable mode satisfies $\alpha(0)=0$. The growth rate $\operatorname{Re}(\alpha)$ is positive for $\nu_{0}(0)=0.05$ for $0<k \leqslant k_{c}$ with $k_{c} \approx 0.007$ (figure [7(a)). The magnification around $k=0$ in figure 8 shows that $\operatorname{Re}(\alpha)$ grows quadratically 
(a)

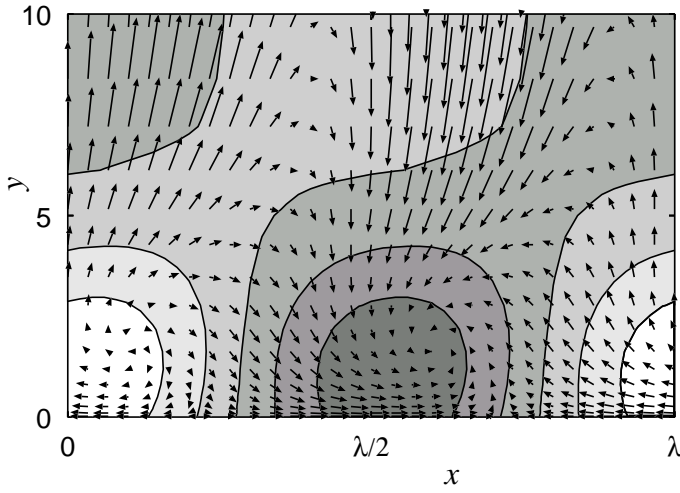

(b)

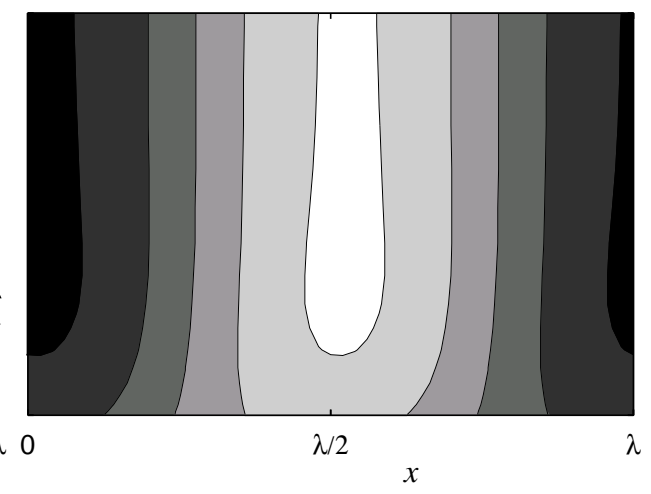

Figure 10. The eigenfunctions of the least stable mode for $\phi=0.05, \theta=16^{\circ}$, and $\nu_{0}(0)=0.10$ (P3 in figure 6), whose wave number is $k=0.002 . \lambda$ is the wavelength of this eigenmode, $\lambda=2 \pi / k$. The contour of the density and the temperature eigenfunctions are shown in (a) and (b), respectively, by grey scale, where the brighter (darker) region corresponds to the larger positive (negative) value. The arrows in (a) indicate the corresponding velocity eigenfunction. It shows that the grains flow from the brighter region into the darker density region, namely, the density perturbation decays. Note that the temperature perturbation is negative at the region of the positive density perturbation.

(a)

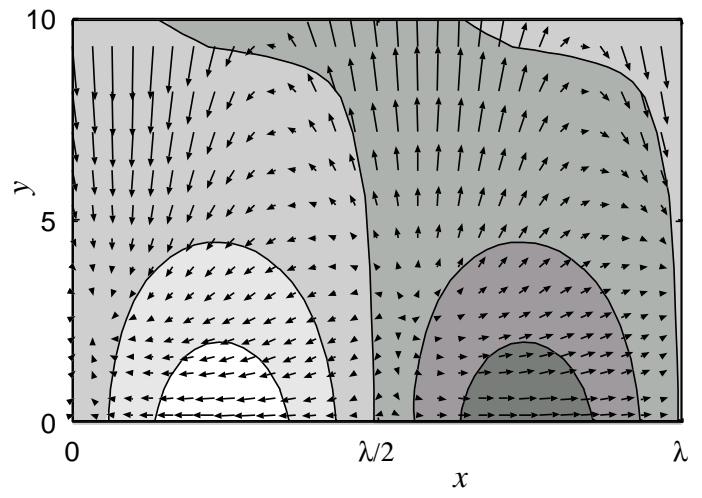

(b)

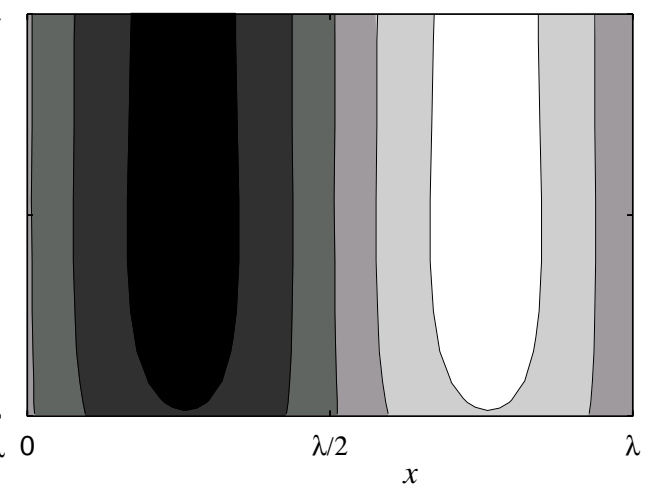

Figure 11. The eigenfunctions of the unstable mode for $\phi=0.05, \theta=16^{\circ}$, and $\nu_{0}(0)=0.05$ (P1 in figure 6), whose wave number is $k=0.002$. The contour of the density eigenfunction and the velocity eigenfunction in (a) show that the grains flow from the region of negative density perturbation into that of positive perturbation, namely, the perturbation is amplified and results in the formation of the density wave. The temperature perturbation in (b) is negative at the region of positive density perturbation.

in $k$ for small $k$. As $\nu_{0}(0)$ is increased, the maximum value of $\operatorname{Re}[\alpha]$ decreases and becomes negative for all $k$ (figure 7 (b) and (c)).

The dispersion relations for the unstable modes at $\mathrm{P} 4$ (in the regime $\mathrm{A}$ ) and at $\mathrm{P} 5$ (in the regime C) are shown in figure 9 for $\left(\theta, \nu_{0}(0)\right)=\left(14^{\circ}, 0.05\right)(\mathrm{a})$ and $\left(17^{\circ}, 0.10\right)(\mathrm{b})$, respectively. The instability occurs for the long-wavelength perturbation, and both of the dispersion relations show that $\alpha(0)=0$ and the growth rate quadratic in $k$ for small $k$, i.e., $\operatorname{Re}[\alpha(k)] \propto k^{2}$; these features are the same as those in the unstable regime B.

The least stable eigenmodes for $\theta=16^{\circ}$ at $k=0.002$ are shown for the two cases: the stable case of $\nu_{0}(0)=0.10$ (P3 in figure 6) in figure 10 and the unstable case 


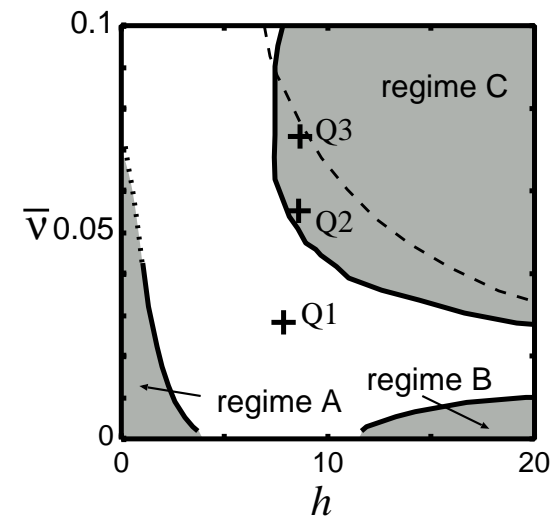

FIgURE 12 . The stability diagram for $h$ vs $\bar{\nu}$ with $\phi=0.10$, where the unstable (stable) regimes are shown by grey (white) regions. We find three unstable regimes $\mathrm{A}, \mathrm{B}$, and $\mathrm{C}$. The dashed line shows the boundary between the region of the non-monotonic density profiles and that of the monotonic density profiles (see also figure 4). The dispersion relations at the points Q1 Q3 are shown in figure 13

of $\nu_{0}(0)=0.05$ in the regime $\mathrm{B}$ (P1 in figure [6) in figure 11] over one wavelength $\lambda=2 \pi / k$. The contours in figures 10(a) and 11(a) show the density eigenfunctions; the brighter (darker) regions indicate the positive (negative) regions, and the arrows represent the corresponding velocity eigenfunctions. In the both figures near $y=0$, we see that the velocity perturbations point to the positive (negative) $x$ direction in the regions of negative (positive) density perturbation. The contours for the corresponding temperature eigenfunctions plotted in figures 10(b) and 11(b) show that the regions where the density perturbation is negative (darker regions in figures 10(a) and 11(a)) roughly correspond to the positive temperature perturbations (brighter regions in figures 10(b) and 11 (b)).

The difference between the stable mode (figure 101) and the unstable mode (figure 11) is seen if we focus on the divergence of velocity perturbation. In the case of the stable mode for $\nu_{0}(0)=0.10$ (figure [10(a)), the grains flow into the region where the density perturbation is negative (see the region around $x \approx \lambda / 2$ and $y \approx 2$ ), thus the density perturbation has negative feedback. On the other hand, in the case of unstable mode for $\nu_{0}(0)=0.05$ (figure 111(a)), the grains flow into the region where the density perturbation is positive (see the region around $x \approx 0$ and $y \approx 3$ ); As a result, the perturbation grows and eventually causes the nonlinear density wave.

The eigenfunctions of the unstable modes at $\mathrm{P} 4$ in the regime $\mathrm{A}$ and at $\mathrm{P} 5$ in the regime $\mathrm{C}$ show some different characteristics from those at $\mathrm{P} 1$ in the regime $\mathrm{B}$. Reflecting the difference in the steady flow between the regimes $\mathrm{A}$ and $\mathrm{C}$ and the regime $\mathrm{B}$, the denser parts of the density eigenfunctions roughly correspond to the part where the velocity fluctuation has the positive component in the direction parallel to the mean flow. For all three regimes, however, the unstable modes show that the grains flow into the region of the positive density perturbation; this suggests that the instability leads to the density wave in the regimes $\mathrm{A}$ and $\mathrm{C}$ as in the regime $\mathrm{B}$.

\subsubsection{The case of $\phi=0.10$}

The stability diagram for $\phi=0.10$ is shown in figure 12 As in the case of $\phi=0.05$, there are three unstable regimes, but the qualitative difference is that the unstable regime $\mathrm{C}$ at the large $h$ and large $\bar{\nu}$ region contains a part of the region of the monotonic density profiles as well as that of the non-monotonic density profiles. The dispersion 
14

(a)

$\operatorname{Re}(\alpha)$

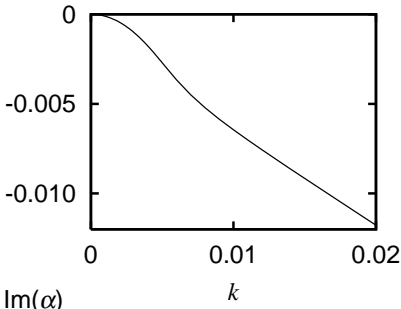

$\operatorname{Im}(\alpha)$

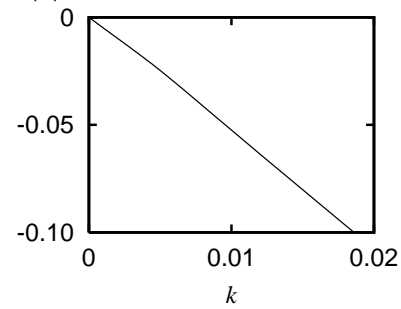

N. Mitarai and H. Nakanishi

(b)

$\operatorname{Re}(\alpha)$

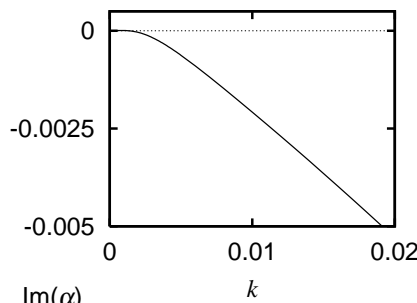

$\operatorname{Im}(\alpha)$

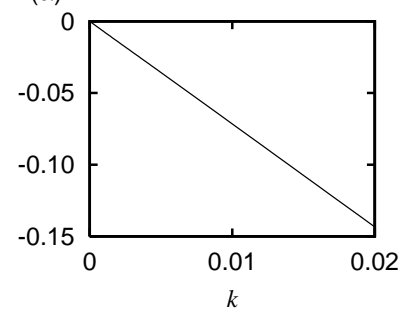

(c)

$\operatorname{Re}(\alpha)$

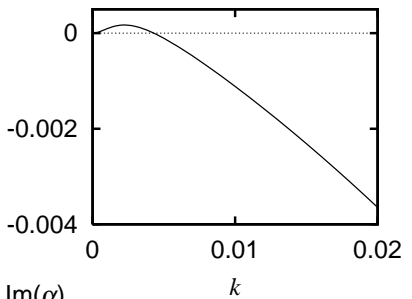

$\operatorname{Im}(\alpha)$

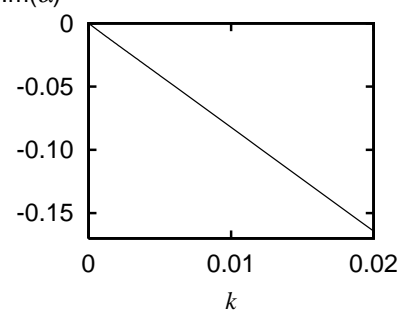

Figure 13. The dispersion relations of the least stable mode with $\phi=0.10$ and $\theta=20^{\circ}$ for $\nu_{0}(0)=0.10$ (a) $0.15(\mathrm{~b})$, and 0.17 (c), which correspond to the points Q1, Q2, and Q3 in figure 12 respectively.

relations around the boundary of the regime $\mathrm{C}$ are shown in figure 13 for $\theta=20^{\circ}$ and $\nu_{0}(0)=0.10(\mathrm{a}), 0.15(\mathrm{~b})$ and $0.17(\mathrm{c})$, which correspond to the points Q1, Q2, and Q3, respectively, in the stability diagram 12 It is seen that the instability occurs against the long-wavelength perturbation.

The unstable eigenmodes for $\theta=20^{\circ}$ and $\nu_{0}(0)=0.17$ (Q3 in figure 12) with $k=0.002$ are shown in figure 14 The density and the velocity eigenfunctions indicate that the grains flow into the the region of positive density perturbation (see the region around $x \approx \lambda / 2, y \approx 0$; the magnification is shown (c)), thus the density perturbation will grow. The difference from the case in figure 111 is that the velocity perturbation at the floor $(y=0)$ is in the positive $x$ direction in the region of the positive density perturbation; namely, the particles flow faster in the region where they get dense.

\section{Discussions}

We have calculated the steady flow solutions and examined their linear stability under the longitudinal perturbation in the cases of $\phi=0.05$ and $\phi=0.10$. The linear stability analysis revealed that there are three unstable regimes $\mathrm{A}, \mathrm{B}$, and $\mathrm{C}$ in the $(h, \bar{\nu})$ plane in both of the cases. The regimes $\mathrm{A}$ and $\mathrm{B}$ are at the small $\bar{\nu}$ region, while the regime $\mathrm{C}$ is at the large $\bar{\nu}$ region. The difference between the regimes $\mathrm{A}$ and $\mathrm{B}$ is the density dependence of the flow velocity; the denser flow is faster in the regime A, while the flow with lower density is faster in the regime $\mathrm{B}$. The regime $\mathrm{C}$ lies within the region where the density profile is non-monotonic in the case of $\phi=0.05$, while the regime $\mathrm{C}$ includes a part of the region of the monotonic density profiles for $\phi=0.10$, although the region of the regime $\mathrm{C}$ roughly corresponds with that of the non-monotonic density profiles. In all the regimes, the dispersion relation of the unstable mode shows the features that (i) $\alpha(0)=0$ and (ii) $\operatorname{Re}[\alpha(k)] \propto k^{2}$ for small $k$. The obtained unstable eigenmodes suggest that the instability causes the density wave.

In this section, we give some discussions on the mechanism of the instability, com- 
(a)

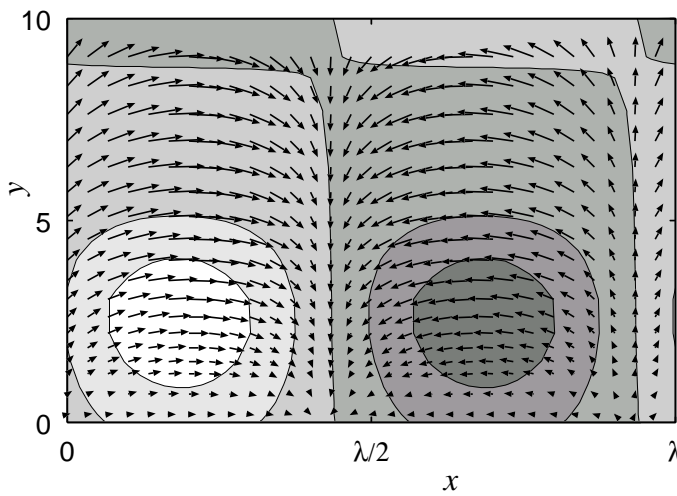

(b)

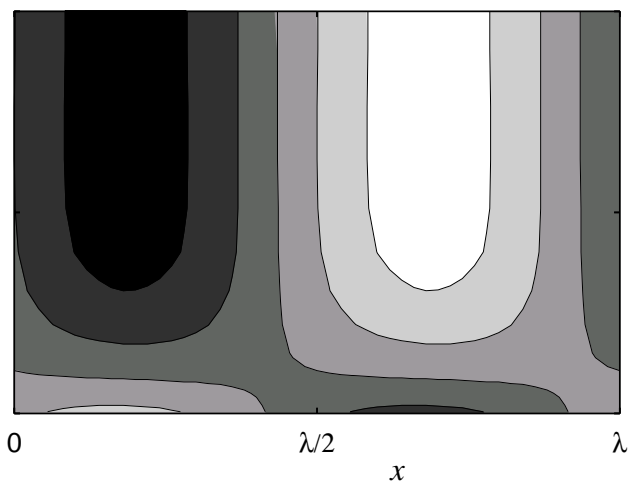

(c)

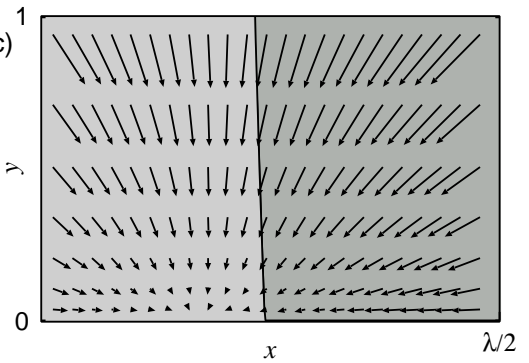

Figure 14. The eigenfunctions of the unstable mode for $\phi=0.10, \theta=20^{\circ}$, and $\nu_{0}(0)=0.17$ (Q3 in figure 12), whose wave number is $k=0.002$. The contour of the density eigenfunction and the velocity eigenfunction in (a) show that the grains flow into the the region of the positive density perturbation. In (b), the contour of the temperature perturbation is shown. (c) is the magnification of (a) around $x \approx \lambda / 2, y \approx 1$.

parison with simulations, and the relationship with the transverse instability studied by Forterre \& Pouliquen(2002)

\subsection{Mechanism of the instability}

\subsubsection{Kinematic wave and the long-wave instability}

The long-wavelength instability which results in the density wave is well known for quasi-one dimensional flows, such as the wave formation in the film flow (Smith 1993; Ooshida 1999), the density wave formation in the granular flow in a narrow vertical pipe (Raafat, Hulin \& Herrmann 1996; Moriyama et al.1998), and the jam formation in the traffic flow (Kerner \& Konhäuser 1993; Bando et al.1995; Mitarai \& Nakanishi 2000a, 2000b). This instability is closely related to the continuity of "density" $\bar{\rho}$ (the thickness in the case of incompressible fluid, the density per unit length along the pipe for the granular flow, or the density of cars for the traffic flow): $\bar{\rho}$ obeys the equation of continuity in the form

$$
\frac{\partial \bar{\rho}(x, t)}{\partial t}+\frac{\partial Q(x, t)}{\partial x}=0
$$

where $Q$ is the flux. The flux $Q$ may be expressed in terms of $\bar{\rho}$ and its spatial derivatives, but may also depend on time especially when the inertia effect exists. In the longwavelength and the long-time limit, the effect of the spatial derivatives of $\bar{\rho}$ and the inertia on $Q$ may be neglected. Then the flux is determined by the density; $Q(x, t)=Q_{0}(\bar{\rho}(x, t))$, 
and equation (5.1) becomes

$$
\frac{\partial \bar{\rho}(x, t)}{\partial t}-c \frac{\partial \bar{\rho}(x, t)}{\partial x}=0 \quad \text { with } \quad c=-\frac{\mathrm{d} Q_{0}(\bar{\rho})}{\mathrm{d} \bar{\rho}} .
$$

The wave that can be described by this equation is called the kinematic wave (Whitham 1974). The effect of the inertia and spatial derivatives for the small but nonzero wavelength appears as the growth rate quadratic in $k$ (Whitham 1974; Ooshida 1999), which causes the instability to yield the density wave. The actual form of the flux $Q$ depends on a physical system, but we call the instability caused through this mechanism "the long-wave instability" after $\operatorname{Smith}(1993)$ Note that the unstable mode caused by the long-wave instability has the following three features for the complex growth rate $\alpha(k)$ : (i) $\alpha(0)=0$, (ii) $\operatorname{Re}[\alpha(k)] \propto k^{2}$ for small $k$, and (iii) the phase velocity of the least stable mode $c=\operatorname{Im}[\alpha(k)] / k$ is given by $-\mathrm{d} Q_{0} / \mathrm{d} \bar{\rho}$ in the long-wavelength limit.

The unstable modes obtained in the present analysis of the two-dimensional slope flow satisfy all of the features (i), (ii) and (iii); the features (i) and (ii) have been already pointed out in the text, and (iii) can be seen in figure 8 (b) in the case of $\phi=0.05$, $\nu_{0}(0)=0.05$, and $\theta=16^{\circ}$. Actually, (iii) can be shown using (i) and the equation of continuity (2.1) as shown in Appendix B.

These features strongly suggest that the longitudinal instability in the slope flow is the long-wave instability of the kinematic wave in a quasi-one dimensional system for all of the regimes $\mathrm{A}, \mathrm{B}$, and $\mathrm{C}$.

One may think that, in the regime $\mathrm{C}$, which lies in the region of the non-monotonic density profile in the case of $\phi=0.05$ (figure [6), the non-monotonic density profile might play a crucial role in the instability as in the case of the transverse instability of Rayleigh-Bérnard type, where the convection occurs due to the non-monotonic density profile (Forterre \& Pouliquen 2002; Carpen \& Brady 2002); however, the fact that the regime $\mathrm{C}$ contains a part of the region of the monotonic density profile in the case of $\phi=0.10$ (figure 12) suggests that the shape of the density profile does not determine the instability. It should be also noted that the present longitudinal instability occurs at the long wavelength while the transverse instability appears at the finite wavelength.

\subsubsection{One dimensional model}

In order to confirm that the longitudinal instability is the long-wave instability of the one-dimensional kinematic wave, we now try to reduce our two-dimensional model into the one-dimensional model that preserve the major features of the original model. In spite of crudeness of our procedure, we will see the obtained one-dimensional model shows roughly the same stability diagram for the long-wave instability of the kinematic wave.

The way we obtain the one-dimensional model is to integrate the original equations in $y$-direction from 0 to $\infty$; the idea is similar to Valance \& Pennec(1998), where the one-dimensional model has been obtained for the flow in a vertical chute by integrating the equations across the chute width.

We define the one-dimensional density $\bar{\rho}(x, t)$, the average velocity $\bar{u}(x, t)$, and the average temperature $\bar{T}(x, t)$ as

$$
\begin{aligned}
\bar{\rho}(x, t) & \equiv \int_{0}^{\infty} \nu(x, y, t) \mathrm{d} y, \\
\bar{\rho}(x, t) \bar{u}(x, t) & \equiv \int_{0}^{\infty} \nu(x, y, t) u(x, y, t) \mathrm{d} y,
\end{aligned}
$$




$$
\bar{\rho}(x, t) \bar{T}(x, t) \equiv \int_{0}^{\infty} \nu(x, y, t) T(x, y, t) \mathrm{d} y .
$$

The one-dimensional equation of continuity obtained by integrating (2.1) is

$$
\partial_{t} \bar{\rho}+\partial_{x}(\bar{\rho} \bar{u})=0
$$

where $\partial_{t}$ and $\partial_{x}$ represent $\partial / \partial t$ and $\partial / \partial x$, respectively. (5.6) is in the form of (5.1) with the flux $Q(x, t)$ given by $Q=\bar{\rho} \bar{u}$.

By integrating the $x$-component of the equation of motion (2.2), we obtain

$$
\begin{aligned}
& \partial_{t}(\bar{\rho} \bar{u})+\partial_{x} \int_{0}^{\infty} \nu(x, y, t) u(x, y, t)^{2} \mathrm{~d} y \\
& =\bar{\rho} \sin \theta+\Sigma_{y x}(\nu(0), T(0), u(0))-\partial_{x}\left(\int_{0}^{\infty} \Sigma_{x x}(\nu, T, u) \mathrm{d} y\right)
\end{aligned}
$$

which determines the evolution of $Q=\bar{\rho} \bar{u}$. The second term in RHS is the shear stress at the floor and comes from the integration of $\partial_{y} \Sigma_{y x}$.

To simplify (5.7), we make the following approximations; (i) Replace the second term in LHS $\int_{0}^{\infty} \nu(x, y, t) u(x, y, t)^{2} \mathrm{~d} y$ by $\bar{\rho} \bar{u}^{2}$, (ii) Neglect the velocity in the $y$-direction, $v$, and use the dilute limit expressions for the the pressure and viscosities in $\Sigma_{x x}$, namely, we replace $\Sigma_{x x}$ by $\nu T-(4 / 3) f_{2}(0) \sqrt{T} \partial_{x} u$, where the first term comes from the pressure and the second term comes from the dilute limit of the shear viscosity. The second viscosity $\zeta(\nu, T)$ is neglected because it is a higher order quantity in $\nu$ (see (2.7)). (iii) Estimate the integration of the shear viscosity term by multiplying the integrand by the decay length of the density $(T / \cos \theta)$, and replacing $T$ by $\bar{T}, u$ by $\bar{u}$, i.e., replace $(4 / 3) f_{2}(0) \int_{0}^{\infty}\left(\sqrt{T} \partial_{x} u\right) \mathrm{d} y$ by $H(\bar{T}) \cdot(4 / 3) f_{2}(0)\left(\sqrt{\bar{T}} \partial_{x} \bar{u}\right)$, where $H(\bar{T})=\bar{T} / \cos \theta$.

Then we obtain the equation

$\partial_{t}(\bar{\rho} \bar{u})+\partial_{x}\left(\bar{\rho} \bar{u}^{2}\right)=\bar{\rho} \sin \theta+\Sigma_{y x}(\nu(0), T(0), u(0))-\partial_{x}(\bar{\rho} \bar{T})+(4 / 3) f_{2}(0) \partial_{x}\left(H(\bar{T}) \sqrt{\bar{T}} \partial_{x} \bar{u}\right)$.

For the shear stress at the floor $\Sigma_{y x}(\nu(0), T(0), u(0))$, we have the boundary condition of momentum balance (2.9), i.e. $\Sigma_{y x}(\nu(0), T(0), u(0))=-\eta^{*}(\nu(0), T(0)) u(0)$.

To close equations (5.6) and (5.8), we need the relation between $(\nu(0), T(0), u(0))$ and $(\bar{\rho}, \bar{T}, \bar{u})$, and the equation for the average temperature $\bar{T}$. We simply assume that $u(0)=\bar{u}$ and $T(0)=\bar{T}$, and we use the empirical relation between $\nu(0)$ and $\bar{\rho}$ for the steady flows; namely, we take $\nu(0)=F(\bar{\rho})$, with the form of $F(\bar{\rho})$ determined from the steady solution obtained numerically for a fixed $\theta$ and different values of $\nu_{0}(0)$ by using $\nu_{0}(0)=F\left(\bar{\rho}_{0}\right)$ with $\bar{\rho}_{0}=\int_{0}^{\infty} \nu_{0}(y) \mathrm{d} y$. We further assume that $\bar{T}$ is also determined by the one-dimensional density $\bar{\rho}$, rather than to use the integrated equation for the temperature. The form of $\bar{T}=\bar{T}(\bar{\rho})$ is determined from the steady flows, namely, we assume $\bar{T}_{0}=\left(1 / \bar{\rho}_{0}\right) \int_{0}^{\infty} \nu_{0}(y) T_{0}(y) \mathrm{d} y=\bar{T}\left(\bar{\rho}_{0}\right)$.

Now we finally obtain the one-dimensional model in the following form;

$$
\begin{aligned}
\partial_{t} \bar{\rho}+\partial_{x}(\bar{\rho} \bar{u})= & 0 \\
\bar{\rho}\left[\partial_{t} \bar{u}+\bar{u} \partial_{x} \bar{u}\right]= & a(\bar{\rho})[U(\bar{\rho})-\bar{u}]-\partial_{x}(\bar{\rho} \bar{T}(\bar{\rho})) \\
& +(4 / 3) f_{2}(0) \partial_{x}\left(H(\bar{T}(\bar{\rho})) \sqrt{\bar{T}(\bar{\rho})} \partial_{x} \bar{u}\right)
\end{aligned}
$$

where

$$
a(\bar{\rho})=\eta^{*}(F(\bar{\rho}), \bar{T}(\bar{\rho})), \quad U(\bar{\rho})=\frac{\bar{\rho} \sin \theta}{a(\bar{\rho})} .
$$

In this model, the steady solution is given by $\bar{\rho}=\rho_{0}=$ const. and $\bar{u}=U\left(\rho_{0}\right)$, and 
the flux of the steady solution is given by $q_{0}=\rho_{0} U\left(\rho_{0}\right)$. The equation (5.10) with (5.11) shows that the velocity $U\left(\rho_{0}\right)$ is determined by the balance between the acceleration by the gravity and the drag force from the floor.

This model is almost the same form as the traffic flow model proposed by Kerner \& Konhäuser(1993) but with the different form of the function $U(\bar{\rho})$. In the traffic flow model, $U(\bar{\rho})$ is often called the "optimal velocity function", which defines the density dependence of the car velocity and is usually a decreasing function. On the other hand, in the case of the granular flow on a slope, $U(\bar{\rho})$ depends on the inclination angle and the boundary condition at the floor, and can take various forms as can be seen in the plot of $\bar{\rho}_{0}$ vs $Q_{0} \approx q_{0}=\bar{\rho}_{0} U\left(\bar{\rho}_{0}\right)$ $\dagger$ in figures 2(b) and 4 (b).

The linear stability analysis of the steady solution can be performed analytically (for the traffic flow model, see e.g. Wada \& Hayakawa 1998). It is easy to show that the instability condition $\operatorname{Re}[\alpha]>0$ yields

$$
\left(a\left(\rho_{0}\right) U^{\prime}\left(\rho_{0}\right)\right)^{2} k^{2}>\left(\frac{a\left(\rho_{0}\right)}{\rho_{0}}+\bar{\mu}\left(\rho_{0}\right) k^{2}\right)^{2}\left(\rho_{0} \bar{T}\left(\rho_{0}\right)\right)^{\prime} k^{2},
$$

where $\bar{\mu}\left(\rho_{0}\right) \equiv(4 / 3) f_{2}(0) H\left(\bar{T}\left(\rho_{0}\right)\right) \sqrt{\bar{T}\left(\rho_{0}\right)}$ and the prime represents the differentiation by $\rho_{0}$. The $k \rightarrow 0$ limit of (5.12) gives the stability criterion,

$$
\left(\rho_{0} U^{\prime}\left(\rho_{0}\right)\right)^{2}>\left(\rho_{0} \bar{T}\left(\rho_{0}\right)\right)^{\prime} .
$$

The explicit form of the long-wavelength expansion of the dispersion relation for the least stable mode is given by

$$
\alpha(k)=-\left(\rho_{0} U\left(\rho_{0}\right)\right)^{\prime} i k+\frac{\rho_{0}}{a\left(\rho_{0}\right)}\left[\left(\rho_{0} U^{\prime}\left(\rho_{0}\right)\right)^{2}-\left(\rho_{0} \bar{T}\left(\rho_{0}\right)\right)^{\prime}\right] k^{2}+O\left(k^{3}\right),
$$

and we see that the phase velocity of this mode in the long-wavelength limit is given by $c=-\left(\rho_{0} U\left(\rho_{0}\right)\right)^{\prime}=-d q_{0} / d \rho_{0}$, which shows that this is the kinematic wave. The instability arises when the coefficient of the $k^{2}$ term becomes positive, which occurs if the change of the velocity against density fluctuation is too fast compared to the effect of the pressure that reduces the density fluctuation. Note that the criterion of the instability (5.13) does not depend on the shear viscosity term $\bar{\mu}\left(\rho_{0}\right)$ because it only appears in the fourth order term in $k$ (see (5.12) ); the approximation for that term does not crucially affect on the criterion.

The stability diagrams for $\phi=0.05$ and $\phi=0.10$ obtained from this one-dimensional model are shown in figures 15. In spite of the crude approximations used in the derivation of the one-dimensional model, the stability diagrams are qualitatively similar to those of the original model. The similarity further indicates that the density wave formation can be understood by the long-wave instability in quasi-one dimensional systems, like in the film flow and the traffic flow.

It should be noticed that, in this one-dimensional model, the effect of the parameters in the original model such as $e_{p}, \phi$, and $\Phi$ are included more or less implicitly in the functional forms of $U(\bar{\rho})$ and $\bar{T}(\bar{\rho})$ ( $\phi$ also appears explicitly in (5.11) through $\eta^{*}$ ), and the $y$-dependences of the variables also affect $U(\bar{\rho})$ and $\bar{T}(\bar{\rho})$ through the integration. Any changes that affect $U$ and $\bar{T}$ result in the changes of the unstable regions (see the criterion (5.13) ), although the nature of the instability remains the same.

Before concluding this subsection, let us make a few comments on the works by Wang, Jackson, \& Sundaresan(1997) and Valance \& Pennec(1998) on the stability anal-

$\dagger$ The flux $q_{0}$ in the one-dimensional model is not exactly the same as the flux $Q_{0}$ in the original two-dimensional flow, due to the approximations used to derive $U(\bar{\rho})$. 

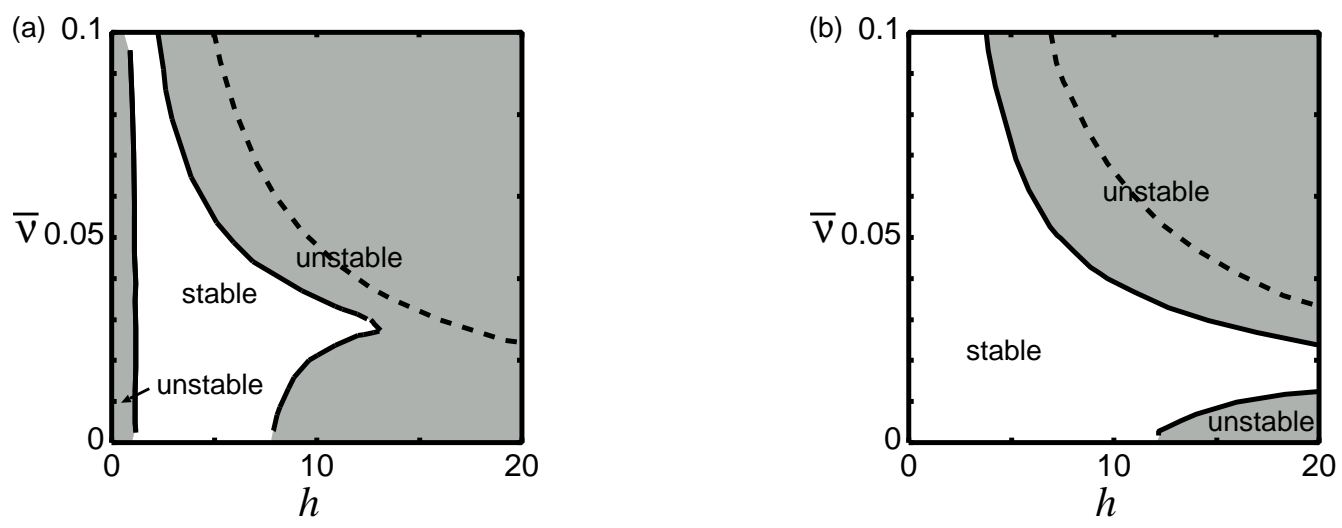

Figure 15. The stability diagram for $(\mathrm{a}) \phi=0.05$ and $(\mathrm{b}) \phi=0.10$ obtained from the one-dimensional model. The unstable (stable) regimes are shown by grey (white) regions. The dashed line shows the boundary between the region of the non-monotonic density profiles and that of the monotonic density profiles for the original model.

ysis of granular flow in a vertical chute using hydrodynamic models of rapid granular flow; Wang et al.(1997) performed the linear stability analysis numerically as in the present work, and Valance \& Pennec(1998) analyzed the density wave by deriving a onedimensional model from hydrodynamic equations for rapid flow.

In the analysis of Wang et al.(1997), a parameter region has been found where the steady flow is unstable against longitudinal long-wavelength perturbation to form the density wave (figure 9 and 10 in Wang et al.1997). This instability might be also understood as the long-wave instability observed in the present analysis. On the other hand, they also found the instabilities for finite wavelength perturbations, which has not been observed here.

The analysis by Valance \& Pennec(1998) shows clear similarity of the instability in the chute flow to the one in the slope flow. The one-dimensional model they obtained (we call it the VP model) has the mathematical structure and physical mechanism similar to those of our one-dimensional model: the velocity of the steady solution is determined by the balance of the gravitational acceleration and the drag force from the wall, and shows the long-wave instability for which the criterion is determined by the change of the velocity against density fluctuation and the pressure term.

These results suggest that the instabilities in the vertical chute flow and the slope flow are in the same class.

\subsection{Comparison with the molecular dynamics simulations}

We find some qualitative agreements between the present results and our previous simulations (Mitarai \& Nakanishi 2001) as follows.

Our simulations were performed for a fixed inclination angle and a particular roughness of the slope with the periodic boundary condition imposed along the flow direction. Within the examined parameter region, the steady flow shows the monotonic density profile (Mitarai, Hayakawa, \& Nakanishi 2002), and the flow with lower density has higher velocity.

It has been demonstrated that the density wave appears only in the long system with low enough particle density. We have performed the simulations for several sets of the slope length $L$ and the particle number $N$. In the case of the particle density $N \sigma / L \approx 1.0$ (single layer), the clear density wave is not formed for $L=250.5 \sigma$ and $L=501 \sigma$, whereas 
the density wave appears for $L=1002 \sigma$. Upon changing the density with a fixed system length $L=501 \sigma$, the density wave is formed when $N \sigma / L \approx 0.75$, while the steady flow is stable for the denser cases with $N \sigma / L \approx 1.0$ and 2.0 .

These tendencies of the simulations agree with the behaviors around the unstable regime $\mathrm{B}$ of the present model on the three points: (i)The flow with lower density is faster, (ii)The flow with lower density is less stable, and (iii) The critical wavelength for instability is very long.

Regarding (iii), the critical wave length $\lambda_{c}=2 \pi / k_{c}$ is much longer than the particle diameter; $\lambda_{c} \approx 900 \sigma$ for $\theta=16^{\circ}$ and $\nu_{0}(0)=0.05$, for example. This seems to be comparable with our simulation results, where the critical slope length $L_{c}$ was between $500 \sigma$ and $1000 \sigma$ for $N \sigma / L \approx 1.0$. We do not understand yet how such a small wave number arises, but we suspect that it comes from the long mean free path in the large $y$ region where the density is low, namely, the particles flying over the clusters for a long distance prevent the growth of clusters in a smaller length scale.

Based on the observations (i), (ii), and (iii), the parameters that we have simulated happens to be in the regime $\mathrm{B}$, but if the simulations are performed with different densities, inclination angles, and/or boundary conditions at the floor, the behavior that corresponds to the regime A or C may be found.

\subsection{Comparison with the stability analysis against the transverse perturbations}

Forterre \& Pouliquen(2002) examined the linear stability of the granular flow on a wide slope against the perturbation transverse to the flowing direction, in order to understand the regular streak pattern along the flow direction observed in their experiments (Forterre $\&$ Pouliqen 2001). They mainly focused on the parameter region where the non-monotonic density profile (or what they call the "inverted density profile") is observed, because they expected that such a flow would be unstable to form the vortex rolls from the analogy to the Rayleigh-Bérnard instability. They have shown that, the flow is unstable against the transverse perturbation in the large part of the parameter region where the inverted density profile is observed. The unstable mode shows the vortex-like pattern, and they concluded that the streaks observed in the experiments result from the rolls of vortices. They also found that the flow with the monotonic density profile becomes unstable for some parameters, but the detail has not been reported.

One of the differences between this transverse Rayleigh-Bérnard type instability and the longitudinal long-wave instability appears in the length scale of the instability. The longitudinal instability occurs against the long-wavelength perturbations in the long-time behavior as can be seen in the dispersion relations, while the transverse instability occurs at a finite length scale comparable with the vortex roll. Our analysis shows that there is a parameter region where both of the instability may occur, around the region of the non-monotonic density profiles (the regime C). It should be interesting to investigate how the two instabilities interfere or not by the full three dimensional analysis.

In the large inclination angle, Forterre \& Pouliquen(2002) also observed the square lattice pattern. This phenomenon cannot be understood by the simple superposition of the long-wave instability and the Rayleigh-Bérnard type instability, because the length scale of the long-wave instability is much longer than the one of the lattice pattern.

\section{Summary}

The steady flows and their linear stability are analyzed for the granular flow on a slope using the hydrodynamic model with the constitutive relations derived from the kinetic 
theory of inelastic spheres. We mainly focused on the relatively low density region where the density decays monotonically.

The stability diagram shows the three unstable regimes $\mathrm{A}, \mathrm{B}$, and $\mathrm{C}$ in the both cases of $\phi=0.05$ and $\phi=0.10$. Two of the unstable regimes A and B are in the lower density region, and the regime $\mathrm{C}$ is in the high density region. The difference between the regimes $\mathrm{A}$ and $\mathrm{B}$ is that, the denser flow is faster in the regime $\mathrm{A}$ at the small $h$ side, while the flow with lower density is faster in the regime $\mathrm{B}$ at the large $h$ side. The regime $\mathrm{C}$ is in the large $h$ and large $\bar{\nu}$ region; it lies within the region of the non-monotonic density profile in the case of $\phi=0.05$, while it contains a part of the region of monotonic density profiles in the case of $\phi=0.10$, although the region of the regime $\mathrm{C}$ roughly corresponds with that of the non-monotonic density profile. In all regimes, the instability occurs for the long-wavelength perturbations and results in the formation of density wave. It has been found that the behaviors around the unstable regime B agree with the tendencies in our previous simulations of density wave formations.

The dispersion of the complex growth rate $\alpha(k)$ has the features that (i) $\alpha(0)=0$, (ii) $\operatorname{Re}[\alpha(k)] \propto k^{2}$ for small $k$, and (iii) $\operatorname{Im}[\alpha(k)] / k=-\mathrm{d} Q_{0} / \mathrm{d} \bar{\rho}_{0}$ in the long-wavelength limit. These strongly suggest that the instability is the long-wave instability of the kinematic waves, which is often found in quasi-one dimensional flows. This is different from the transverse instability studied by Forterre \& Pouliquen(2002), where the flow is unstable at the finite wave number.

In order to confirm that the instability is the long-wave instability of the kinematic wave, we simplified the original equations rather heuristically into a one-dimensional model, and showed that the long-wave instability occurs in the derived one-dimensional model. The stability diagram obtained from the one-dimensional model corresponds qualitatively to the one obtained from the original equations.

N. M. is grateful to Ooshida Takeshi for informative discussions. This work was partially supported by Hosokawa powder technology foundation and Grant-in-Aid for JSPS fellows.

\section{Appendix A. Linearized equations}

In this appendix, the linearized governing equations for the longitudinal perturbations (4.1)-(4.3) and the boundary conditions are given (Forterre \& Pouliquen 2002, see also Alam \& Nott 1998). The superscript 0 denotes that the quantities are for steady solution. The subscript $\nu$ and $T$ denotes the partial derivatives by the variables; $p_{\nu}^{0}=$ $\partial p(\nu, T) /\left.\partial \nu\right|_{\nu=\nu_{0}, T=T_{0}}$. The subscript $y$ indicates the total differential with respect to $y$, namely, $p_{y}^{0}=\mathrm{d} p^{0}(\nu(y), T(y)) / \mathrm{d} y=\left[\partial p(\nu, T) /\left.\partial \nu\right|_{\nu=\nu_{0}, T=T_{0}}\right] \nu_{0, y}+\left[\partial p(\nu, T) /\left.\partial T\right|_{\nu=\nu_{0}, T=T_{0}}\right] T_{0, y}$, $\nu_{0, y}=\mathrm{d} \nu_{0}(y) / \mathrm{d} y$, and so on. The expressions of $p(\nu, T), \mu(\nu, T), \zeta(\nu, T), \kappa(\nu, T)$, and $\Gamma(\nu, T)$ are given in (2.7), and $\xi(\nu, T)=\zeta-2 \mu / 3$. Then, by inserting (4.1)-(4.3) into (2.1)-(2.3) through (3.1)-(3.3), we obtain the following expressions;

$$
\begin{aligned}
& {\left[\partial_{t}+u_{0} \partial_{x}\right] \nu_{1}+\left[\nu_{0} \partial_{x}\right] u_{1}+\left[\nu_{0, y}+\nu_{0} \partial_{y}\right] v_{1}=0, } \\
& {\left[\sin \theta-p_{\nu}^{0} \partial_{x}+u_{0, y y} \mu_{\nu}^{0}+u_{0, y} \mu_{\nu, y}^{0}+u_{0, y} \mu_{\nu}^{0} \partial_{y}\right] \nu_{1} } \\
+ & {\left[-\nu_{0} \partial_{t}-\nu_{0} u_{0} \partial_{x}+\left(\xi^{0}+2 \mu^{0}\right) \partial_{x}^{2}+\mu_{y}^{0} \partial_{y}+\mu^{0} \partial_{y}^{2}\right] u_{1} } \\
+ & {\left[-\nu_{0} u_{0, y}+\mu_{y}^{0} \partial_{x}+\left(\xi^{0}+\mu_{0}\right) \partial_{x} \partial_{y}\right] v_{1} } \\
+ & {\left[-p_{T}^{0} \partial_{x}+u_{0, y y} \mu_{T}^{0}+u_{0, y} \mu_{T y}^{0}+u_{0, y} \mu_{T}^{0} \partial_{y}\right] T_{1}=0, } \\
& {\left[-\cos \theta-p_{\nu y}^{0}-p_{\nu}^{0} \partial_{y}+u_{0, y} \mu_{\nu}^{0} \partial_{x}\right] \nu_{1} }
\end{aligned}
$$




$$
\begin{aligned}
+ & {\left[\xi_{y}^{0} \partial_{x}+\xi^{0} \partial_{x} \partial_{y}+\mu^{0} \partial_{x} \partial_{y}\right] u_{1} } \\
+ & {\left[-\nu_{0} \partial_{t}-\nu_{0} u_{0} \partial_{x}+\xi_{y}^{0} \partial_{y}+\xi^{0} \partial_{y}^{2}+2 \mu_{y}^{0} \partial_{y}+2 \mu_{0} \partial_{y}^{2}+\mu_{0} \partial_{x}^{2}\right] v_{1} } \\
+ & {\left[-p_{T y}^{0}-p_{T}^{0} \partial_{y}+u_{0, y} \mu_{T}^{0} \partial_{x}\right] T_{1}=0, } \\
& {\left[\kappa_{\nu y}^{0} T_{0, y}+\kappa_{\nu}^{0} T_{0, y y}+\kappa_{\nu}^{0} T_{0, y} \partial_{y}-\Gamma_{\nu}^{0}+u_{0, y}^{2} \mu_{\nu}^{0}\right] \nu_{1} } \\
+ & {\left[-p^{0} \partial_{x}+2 \mu^{0} u_{0, y} \partial_{y}\right] u_{1} } \\
+ & {\left[-\frac{3}{2} \nu_{0} T_{0, y}-p^{0} \partial_{y}+2 \mu^{0} u_{0, y} \partial_{x}\right] v_{1} } \\
+ & {\left[-\frac{3}{2} \nu_{0} \partial_{t}-\frac{3}{2} \nu_{0} u_{0} \partial_{x}+\kappa^{0} \partial_{x}^{2}+\kappa_{y}^{0} \partial_{y}+\kappa^{0} \partial_{y}^{2}\right.} \\
& \left.+\kappa_{T y}^{0} T_{0, y}+\kappa_{T}^{0} T_{0, y y}+\kappa_{T}^{0} T_{0, y} \partial_{y}-\Gamma_{T}^{0}+u_{0, y}^{2} \mu_{T}^{0}\right] T_{1}=0 .
\end{aligned}
$$

The linearized boundary conditions at $y=0$ are obtained from (2.9) and (2.10) as

$$
\begin{aligned}
& \partial_{y} u_{1}=\phi\left[u_{0} f_{7, \nu}^{0} \nu_{1}+f_{7}^{0} u_{1}\right], \\
& \partial_{y} T_{1}=-f_{6, \nu}^{0}\left[\frac{1}{3} \phi u_{0}^{2}-\frac{1}{2} \Phi T_{0}\right] \nu_{1}-f_{6}^{0}\left[\frac{2}{3} \phi u_{0} u_{1}-\frac{1}{2} \Phi T_{1}\right] .
\end{aligned}
$$

\section{Appendix B. The long-wavelength limit of the phase velocity}

We have obtained the dispersion relation of the least stable mode, $\alpha=\alpha(k)$, and numerically found that it satisfies

$$
\alpha(0)=0 .
$$

In this appendix, we show that the phase velocity of this mode, $c=\operatorname{Im}(\alpha(k)) / k$, satisfies

$$
c=-\frac{\mathrm{d} Q_{0}}{\mathrm{~d} \bar{\rho}_{0}}
$$

in the long-wavelength limit $(k \rightarrow 0)$, which suggests the mode is the kinematic wave. Here, $\bar{\rho}_{0}$ and $Q_{0}$ are the one-dimensional density and the flux of the steady solution, respectively, defined in (3.15).

Note that the derivative $\mathrm{d} Q_{0} / \mathrm{d} \bar{\rho}_{0}$ is taken within a family of solutions for a fixed inclination angle as below. For a given inclination angle, the density at the floor $\nu_{0}(0)=\beta$ is a continuous parameter to specify a steady solution. To express $\beta$ dependence explicitly, we rewrite the steady solutions as

$$
\begin{aligned}
\nu(x, y, z, t) & =\nu_{0}(y ; \beta), \\
\boldsymbol{u}(x, y, z, t) & =\left(u_{0}(y ; \beta), 0,0\right), \\
T(x, y, z, t) & =T_{0}(y ; \beta) .
\end{aligned}
$$

Then $d Q_{0} / d \bar{\rho}_{0}$ is given by

$$
\frac{\mathrm{d} Q_{0}}{\mathrm{~d} \bar{\rho}_{0}}=\frac{\mathrm{d} Q_{0}(\beta) / \mathrm{d} \beta}{\mathrm{d} \bar{\rho}_{0}(\beta) / \mathrm{d} \beta}=\frac{\int_{0}^{\infty}\left[\partial\left(\nu_{0}(y ; \beta) u_{0}(y ; \beta)\right) / \partial \beta\right] \mathrm{d} y}{\int_{0}^{\infty}\left[\partial \nu_{0}(y ; \beta) / \partial \beta\right] \mathrm{d} y} .
$$

In order to show (B2), let us first express $c$ by eigenfunctions of the mode. By linearization of the equation of continuity (2.1) using (4.4), we obtain the following expression:

$$
\alpha \hat{\nu}(y)=-i k\left(\nu_{0}(y ; \beta) \hat{u}(y)+u_{0}(y ; \beta) \hat{\nu}(y)\right)-\partial_{y}\left(\nu_{0}(y ; \beta) \hat{v}(y)\right) .
$$


Integrating (B 7) from $y=0$ to $\infty$, we have

$$
\alpha \int_{0}^{\infty} \hat{\nu}(y) \mathrm{d} y=-i k \int_{0}^{\infty}\left[\nu_{0}(y ; \beta) \hat{u}(y)+u_{0}(y ; \beta) \hat{\nu}(y)\right] \mathrm{d} y .
$$

Here, $\hat{\nu}(y), \hat{u}(y), \hat{v}(y)$, and $\hat{T}(y)$ depend on the wavenumber $k$, and we expands these functions with respect to $k$, i.e.,

$$
\begin{array}{ll}
\hat{\nu}(y)=\hat{\nu}_{0}(y)+i k \hat{\nu}_{1}(y)+\ldots ., \quad \hat{u}(y)=\hat{u}_{0}(y)+i k \hat{u}_{1}(y)+\ldots . \\
\hat{v}(y)=\hat{v}_{0}(y)+i k \hat{v}_{1}(y)+\ldots ., \quad \hat{T}(y)=\hat{T}_{0}(y)+i k \hat{T}_{1}(y)+\ldots,
\end{array}
$$

where $\left(\hat{\nu}_{i}(y), \hat{u}_{i}(y), \hat{v}_{i}(y), \hat{T}_{i}(y)\right)$ do not contain $k$. The long-wavelength expansion of the dispersion relation $\alpha=\alpha(k)$ which satisfies $\alpha(0)=0$ is obtained from (B8) when $\int_{0}^{\infty} \hat{\nu}_{0}(y) \mathrm{d} y \neq 0$, and we have

$$
\alpha(k)=-i k \frac{\int_{0}^{\infty}\left[\nu_{0}(y ; \beta) \hat{u}_{0}(y)+u_{0}(y ; \beta) \hat{\nu}_{0}(y)\right] \mathrm{d} y}{\int_{0}^{\infty} \hat{\nu}_{0}(y) \mathrm{d} y}+O\left(k^{2}\right) .
$$

Namely, $c$ is given by

$$
c=-\frac{\int_{0}^{\infty}\left[\nu_{0}(y ; \beta) \hat{u}_{0}(y)+u_{0}(y ; \beta) \hat{\nu}_{0}(y)\right] \mathrm{d} y}{\int_{0}^{\infty} \hat{\nu}_{0}(y) \mathrm{d} y}
$$

in the long-wavelength limit.

Now all we have to do is to express $\overrightarrow{\hat{X}}_{0} \equiv\left(\hat{\nu}_{0}, \hat{u}_{0}, \hat{v}_{0}, \hat{T}_{0}\right)$ with respect to the steady solution $\vec{X}_{0}(y ; \beta) \equiv\left(\nu_{0}(y ; \beta), u_{0}(y ; \beta), 0, T_{0}(y ; \beta)\right)$. Let us write (2.1)-(2.3) and the boundary conditions in the matrix form:

$$
B \frac{\partial \vec{X}}{\partial t}=\vec{N}(\vec{X}) .
$$

Here, $\vec{X}=(\nu, u, v, T), B$ is a constant matrix, and $\vec{N}$ is a nonlinear operator. A steady solution $\vec{X}_{0}(y ; \beta)$ satisfies

$$
\vec{N}\left(\vec{X}_{0}(y ; \beta)\right)=0,
$$

therefore, from (4.4), $\overrightarrow{\hat{X}}=(\hat{\nu}, \hat{u}, \hat{v}, \hat{T})$ satisfies

$$
\left.\frac{\partial \vec{N}(\vec{X}))}{\partial \vec{X}}\right|_{\vec{X}=\vec{X}_{0}(y ; \beta)} \overrightarrow{\hat{X}}=\alpha(k) B \overrightarrow{\hat{X}}
$$

and expanding (B15) by $k$ with $\alpha(0)=0$, we have

$$
\left.\frac{\partial \vec{N}(\vec{X}))}{\partial \vec{X}}\right|_{\vec{X}=\vec{X}_{0}(y ; \beta)} \vec{X}_{0}=0
$$

for the lowest order of $k$. On the other hand, differentiating (B14) by $\beta$, we obtain

$$
\left.\frac{\partial \vec{N}(\vec{X}))}{\partial \vec{X}}\right|_{\vec{X}=\vec{X}_{0}(y ; \beta)} \frac{\partial \vec{X}_{0}(y ; \beta)}{\partial \beta}=0 .
$$

It is plausible that the mode of zero eigenvalue for $k=0$ does not degenerate, because the mass is the only one conserved quantity (the momentum is lost at the floor, and the energy is dissipated.). Thus, from (B16) and (B17), we obtain $\overrightarrow{\hat{X}}_{0} \propto \partial \vec{X}_{0}(y ; \beta) / \partial \beta$, or more explicitly,

$$
\left(\hat{\nu}_{0}, \hat{u}_{0}, \hat{v}_{0}, \hat{T}_{0}\right) \propto\left(\frac{\partial \nu_{0}(y ; \beta)}{\partial \beta}, \frac{\partial u_{0}(y ; \beta)}{\partial \beta}, 0, \frac{\partial T_{0}(y ; \beta)}{\partial \beta}\right) .
$$


Using (B 12) and (B 18), we get

$$
c=-\frac{\int_{0}^{\infty}\left[\nu_{0}(y ; \beta)\left(\partial u_{0}(y ; \beta) / \partial \beta\right)+u_{0}(y ; \beta)\left(\partial \nu_{0}(y ; \beta) / \partial \beta\right)\right] \mathrm{d} y}{\int_{0}^{\infty}\left(\partial \nu_{0}(y ; \beta) / \partial \beta\right) \mathrm{d} y},
$$

and by comparing this with $(\underline{B} 6)$, we have

$$
c=-\frac{\mathrm{d} Q_{0} / \mathrm{d} \beta}{\mathrm{d} \bar{\rho}_{0} / \mathrm{d} \beta}=-\frac{\mathrm{d} Q_{0}}{\mathrm{~d} \bar{\rho}_{0}}
$$

which is (B2).

\section{REFERENCES}

Ahn, H., Brennen, C. E. \& SAbersky, R. H. 1992 Analysis of the fully developed chute flow of granular materials. J. Appl. Mech. 59, 109-119.

Alam, M. \& Notт, P. R. 1998 Stability of plane Couette flow of a granular material. J. Fluid Mech. 377, 99-136.

Anderson, E., Bai, Z., Bischof, C., Blackford, S., Demmel, J., Dongarra, J., Du Croz, J., Greenbaum, A., Hammarling, S., McKenney, A. \& Sorensen, D. 1999 LAPACK Users' Guide, 3rd edn. Philadelphia, PA: Society for Industrial and Applied Mathematics.

Anderson, K. G. \& JACKsOn, R. 1992 A comparison of the solutions of some proposed equations of motion of granular materials for fully developed flow down inclined planes. J. Fluid Mech. 241, 145-168.

Bando, M., Hasebe, K., Nakayama, A., Shibata, A., \& Sugiyama, A. 1995 Dynamical model of traffic congestion and numerical simulation. Phys. Rev. E 51, 1035-1042.

Boyd, J. P. 2001 Chebyshev and Foulier Spectral Methods (Second Edition). Dover.

Campbell, C. S. 1990 Rapid granular flows. Ann. Rev. Fluid Mech. 22, 57-92.

Canuto, C., Hussaini, M. Y., Quarteroni, A. \& Zang, T. A. 1988 Spectral Methods in Fluid Dynamics. Springer Series in computational physics.

Carpen, I. C. \& Brady, J. F. 2002 Gravitational instability in suspension flow. J. Fluid Mech. 472, 201-210.

Cundall, P. A. \& Strack, O. D. L. 1979 A discrete numerical model for granular assemblies. Geotechnique 29, 47-65.

Forterre, Y. \& Pouliquen, O. 2001 Longitudinal vortices in granular flows. Phys. Rev. Lett. 86, 5886-5889.

Forterre, Y. \& Pouliquen, O. 2002 Stability analysis of rapid granular chute flows: formation of longitudinal vortices. J. Fluid Mech. 467, 361-387.

Goldhirsh, I 2003 Rapid granular flows. Annu. Rev. Fluid Mech. 35 267-293.

Gottilieb, D., Hussaini, M. Y. \& Orsag, S. A. 1984 Theory and applications of spectral methods. In Spectral Methods for Partial Differential Equations (ed. R. G. Voigt, D. Gottilieb \& M. Y. Hussaini). SIAM.

Jaeger, H. M., Nagel, S. R. \& Behringer, R. P. 1996 Granular solids, liquids, and gases. Rev. Mod. Phys. 68, 1259-1273.

Jenkins, J. T. 1992 Boundary conditions for rapid granular flow: flat, frictional walls. J. Appl. Mech. 59, 120-127.

Jenkins, J. T. \& Richman, M. W. 1986 Boundary conditions for plane flows of smooth, nearly elastic, circular disks. J. Fluid Mech. 171, 53-69.

Jenkins, J. T. \& Savage, S. B. 1983 A theory for the rapid flow of identical, smooth, nearly elastic, spherical particles. J. Fluid Mech. 130, 187-202.

Johnson, P. C. \& JACKSON, R. 1987 Frictional-collisional constitutive relations for granular materials, with application to plane shearing. J. Fluid Mech. 176, 67-93.

Johnson, P. C., Nott, P. \& JACkson, R. 1990 Frictional-collisional equations of motion for particulate flows and their application to chutes. J. Fluid Mech. 210, 501-535.

Kerner, B. S. \& KonhäUser, P. 1993 Cluster effect in initially homogeneous traffic flow. Phys. Rev. E 48, R2335-R2338. 
Louge, M. Y. \& Keast, S. C. 2001 On dense granular flows down flat frictional inclines. Phys. Fluids 13, 1213-1233.

Lun, C. K. K. \& Savage, S. B. 1986 The effects of an impact velocity dependent coefficient of restitution on stresses developed by sheared granular materials. Acta Mech. 63, 15-44.

Lun, C. K. K., Savage, S. B., Jeffrey, D. J. \& Chepurniy, N. 1984 Kinetic theories for granular flow: inelastic particles in Couette flow and slightly inelastic particles in a general flowfield. J. Fluid Mech. 223, 223-256.

Malik, M. R. 1990 Numerical methods for hypersonic boundary layer stability. J. Comput. Phys. 86, 376-413.

Mayer, E. W. \& Powell, K. G. 1992 Viscous and inviscid instabilities of a trailing vortex. J. Fluid Mech. 245, 91-114.

Mitarai, N., Hayakawa, H. \& Nakanishi, H. 2002 Collisional granular flow as a micropolar fluid. Phys. Rev. Lett. 88, 174301.

Mitarai, N. \& NAKAnishi, H. $2000 a$ Convective instability and structure formation in traffic flow. J. Phys. Soc. Jpn 69, 3752-3761.

Mitarai, N. \& NAKAnishi, H. $2000 b$ Spatiotemporal structure of traffic flow in a system with an open boundary. Phys. Rev. Lett. 85, 1766-1769.

Mitarai, N. \& NAKAnishi, H. 2001 Instability of dilute granular flows on rough slope. J. Phys. Soc. Jpn. 70, 2809-2812.

Mitarai, N. \& NAKANishi, H. 2003 Hard-sphere limit of soft-sphere model for granular materials: Stiffness dependence of steady granular flow. Phys. Rev. E 67, 021301.

Moriyama, O., Kuroiwa, N., Matsushita, M. \& Hayakawa, H. 1998 4/3 law of granular particles flowing through a vertical pipe. Phys. Rev. Lett. 80, 2833-2836.

OoshidA, T. 1999 Surface equation of falling film flows with moderate Reynolds number and large but finite Weber number. Phys. Fluids 11, 3247-3269.

Pouliquen, O. 1999 Scaling laws in granular flows down rough inclined planes. Phys. Fluids 11, $542-545$.

Prasad, S. N., Pal, D. \& Römkens, M. J. M. 2000 Wave formation on a shallow layer of flowing grains. J. Fluid Mech. 413, 89-110.

RaAfat, T., Hulin, J. P. \& Herrmann, H. J. 1996 Density waves in dry granular media falling through a vertical pipe. Phys. Rev. E 53, 4345-4350.

Richman, M. W. 1988 Boundary conditions based upon a modified Maxwellian velocity distribution for flows of identical, smooth, nearly elastic spheres. Acta Mechanica 75, 227-240.

Savage, S. B. 1984 The mechanics of rapid granular flows. Adv. Appl. Mech. 24, 289-366.

Sмiтн, M.K. 199 The mechanism for the long-wave instability in thin liquid films. J. Fluid Mech. 217, 469-485.

Valance, A. \& Pennec, T. L. 1998 Nonlinear dynamics of density waves in granular flows through narrow vertical channels. Euro. Phys. J. B 5, 223-229.

WANG, C., JACKSON, R. \& Sundaresan S. 1997 Instabilities of fully developed rapid flow of a granular material in a channel. J. Fluid Mech. 342, 179-197.

Wada, S. \& Hayakawa, H. 1998 Kink solution in a fluid model of traffic flow. J. Phys. Soc. Jpn. 67, 763-766.

Whithaм, G.B. 1974 Linear and nonlinear waves. John Wiley \& Sons. 